\title{
IL-2 induces a WAVE2-dependent pathway for actin reorganization that enables WASp-independent human NK cell function
}

\author{
Jordan S. Orange, ${ }^{1,2}$ Sumita Roy-Ghanta, ${ }^{1}$ Emily M. Mace, ${ }^{1}$ Saumya Maru, ${ }^{1}$ Gregory D. Rak, \\ Keri B. Sanborn, ${ }^{1}$ Anders Fasth, ${ }^{3}$ Rushani Saltzman, ${ }^{1}$ Allison Paisley, ${ }^{1}$ \\ Linda Monaco-Shawver, ${ }^{1}$ Pinaki P. Banerjee, ${ }^{1}$ and Rahul Pandey ${ }^{1}$ \\ ${ }^{1}$ Children's Hospital of Philadelphia Research Institute, Philadelphia, Pennsylvania, USA. ²University of Pennsylvania School of Medicine,
Philadelphia, Pennsylvania, USA. ${ }^{3}$ Department of Pediatrics, University of Gothenburg, Gothenburg, Sweden.
}

\begin{abstract}
Wiskott-Aldrich syndrome (WAS) is a primary immunodeficiency associated with an increased susceptibility to herpesvirus infection and hematologic malignancy as well as a deficiency of NK cell function. It is caused by defective WAS protein (WASp). WASp facilitates filamentous actin (F-actin) branching and is required for F-actin accumulation at the NK cell immunological synapse and NK cell cytotoxicity ex vivo. Importantly, the function of WASp-deficient NK cells can be restored in vitro after exposure to IL-2, but the mechanisms underlying this remain unknown. Using a WASp inhibitor as well as cells from patients with WAS, we have defined a direct effect of IL-2 signaling upon F-actin that is independent of WASp function. We found that IL-2 treatment of a patient with WAS enhanced the cytotoxicity of their NK cells and the F-actin content at the immunological synapses formed by their NK cells. IL-2 stimulation of NK cells in vitro activated the WASP homolog WAVE2, which was required for inducing WASp-independent NK cell function, but not for baseline activity. Thus, WAVE2 and WASp define parallel pathways to F-actin reorganization and function in human NK cells; although WAVE2 was not required for NK cell innate function, it was accessible through adaptive immunity via IL-2. These results demonstrate how overlapping cytoskeletal activities can utilize immunologically distinct pathways to achieve synonymous immune function.
\end{abstract}

\section{Introduction}

Wiskott-Aldrich syndrome (WAS) is an X-linked congenital human immunodeficiency characterized by the triad of susceptibility to infection, bleeding, and dermatitis. The gene mutated in WAS encodes the WAS protein (WASp), which is preferentially expressed in hematopoietic cells and facilitates reorganization of the actin cytoskeleton. The WASp C terminus contains binding domains for monomeric $(G)$ actin as well as the actin branching complex Arp2/3. WASp binds G-actin via a verprolin homology $(\mathrm{V})$ region and Arp $2 / 3$ via an acidic (A) region. When Arp2/3 and G-actin are approximated by WASp, the complex can enable formation of a branch point on an existing actin filament. The branching of filamentous actin (F-actin) allows cells to reorganize their cortex to promote subcellular and cellular motility as well as signaling needed for function. WASp serves as a critical regulator of F-actin reorganization in that it exists in an autoinhibited confirmation that prevents its ability to facilitate Arp2/3 function. After cellular activation, however, the conformation of WASp is altered to allow the approximation of Arp2/3 and G-actin at an existing actin filament, thereby enabling actin branching function.

WASp shares actin branching function with a family of proteins. Many have structural similarity to WASp and contain a C-terminal $\mathrm{V}$ and A region. While WASp is preferentially expressed in hematopoietic cells, other family members have critical functions in immune cells. An important example is the WASp family member 2 ,

Authorship note: Sumita Roy-Ghanta and Rahul Pandey contributed equally to this work.

Conflict of interest: The authors have declared that no conflict of interest exists. Citation for this article: J Clin Invest. 2011;121(4):1535-1548. doi:10.1172/JCI44862. also known as WASp family verprolin-homologous 2 (WAVE2). A number of studies have defined critical roles for WAVE2 in T cells, in which it is required for F-actin reorganization and access to cell function $(1,2)$. Although WASp can support actin reorganization in T cells $(3,4)$, WAVE2 may be the critical facilitator. Patients with WAS, however, have been defined as having a number of $\mathrm{T}$ cell abnormalities including abnormal cell surface ruffling (5) as well as a decreased ability to proliferate and produce IL-2 (6-9).

Despite the existence of multiple WASp family members, the clinical immunodeficiency in WAS is wide ranging and is associated with diverse immune abnormalities. Unusual features of the disease include susceptibility to clinically relevant infections with herpesviruses (including severe cases) in approximately one-third of patients as well as hematologic malignancy representing a leading cause of death (10). This suggests a potential insufficiency in NK cell functions, since they are important for human defense against herpesviruses $(11,12)$ and surveillance for malignancy (13). NK cells are best known for their ability to mediate cytotoxicity after ligation of germline-encoded activating receptors. This requires the establishment of a contact between the NK cell and its target followed by the directed secretion of cytolytic molecules contained in specialized organelles called lytic granules. The contact between the NK cell and susceptible target cell is a specialized form of the immunological synapse (IS) known as the NK cell lytic IS, which progresses through a number of individual subcellular steps to facilitate cytotoxicity (14). A relatively early step in the formation of the NK cell lytic IS is the synaptic accumulation of F-actin, which is required for many subsequent steps including clustering of certain cell-surface receptors at and polarization of lytic granules to the IS (15). In human NK cells, WASp accumulates and is required for effective actin reorganization at the synapse (16). 
Resting NK cells from patients with WAS are deficient in NK cell cytotoxicity and do not form an appropriate IS (15-17). In particular, F-actin accumulation at, and lytic granule polarization to, the IS are decreased. The defect in cytotoxicity is present in NK cells from patients who have a WASP mutation leading to absent protein expression as well as from patients with milder disease caused by mutations leading to decreased WASp expression (17). Even these more mildly affected patients, however, have clinical susceptibility to hematologic malignancy (18), thus furthering consideration of a linkage from the clinical phenotype to a deficiency of NK cell function.

Interestingly, the defect in WAS patient NK cell cytotoxicity is only found when cells are tested directly ex vivo and is not present after in vitro culture $(16,17)$. IL-2, which is present in many NK cell culture systems, can restore NK cell cytotoxicity against K562 target cells when added in vitro to WAS patient PBMCs (17). This enhancing effect was found in WAS patients having either severe or mild WASP mutations and has been defined in multiple unrelated individuals (17). Since T cells from WAS patients have a decreased ability to produce IL-2 after specific types of stimulation $(6,7,19)$, it is plausible that WAS NK cells do not have access to sufficient IL-2 in vivo and cannot therefore take advantage of this effect. In the present work, we sought to define the mechanism of how IL-2 might circumvent WASp as well as determine whether there are parallel pathways to actin reorganization that can be differentially accessed in NK cells. We also attempted to determine whether this in vitro phenomenon has relevance in vivo in WAS and designed a clinical trial of IL-2 administration. The goal of this latter effort is ultimately to increase NK cell function to provide additional surveillance against malignancy and defense against herpesviruses. We report that IL-2 can directly induce F-actin reorganization in NK cells and can circumvent WASp by activating WAVE2. We also show that IL-2 can increase NK cell function and F-actin content in vivo in WAS.

\section{Results}

IL-2 induces F-actin content in NK cells independently of target cells. Short-term exposure of NK cells from WAS patients to IL-2 in vitro restores cytotoxicity and enables F-actin accumulation at the lytic IS (17). This suggested that IL-2 could provide the activation signal that would be otherwise defective in resting WAS patient NK cells upon encountering a target cell. Conversely, this result could suggest that IL-2 has a direct effect upon F-actin reorganization in NK cells independently of activation signals provided by target cells. To evaluate this directly, NK cells were incubated in IL-2 in the absence of target cells, fixed, permeabilized, and F-actin content measured by fluorescent phalloidin binding via flow cytometry. This was first evaluated in 2 different immortalized NK cell lines: YTS, which is IL-2 independent for growth, but IL-2 responsive (20); and NK92, which is IL-2 dependent (21). Short-term addition of IL-2 to each of these cell lines increased F-actin content (Figure 1A). To be certain that this effect was not specific to the NK cell lines, PBMCs or purified ex vivo NK cells were prepared and exposed to IL-2 in vitro. With either cell preparation, increased phalloidin fluorescence was detected by flow cytometry, demonstrating that IL-2 can increase F-actin content in ex vivo cells. The concentrations of IL- 2 used in these experiments were derived from dose titration experiments (data not shown). The time that the cells were incubated with IL-2 was also evaluat- ed, and in the NK cell lines as well as PBMCs and ex vivo NK cells, reproducible increases in $\mathrm{F}$-actin content were identified within 2 hours (Figure 1B). Thus, IL-2 induced rapid increases in F-actin when added to NK cells independently of target cells, suggesting a direct effect of the cytokine.

Since our experiments measured F-actin content using fixed cells through indirect visualization with phalloidin, we next evaluated actin distribution directly in live YTS cells stably transduced with GFP-actin. While GFP fluorescence in these cells demonstrates actin and not necessarily filaments, redistribution of fluorescence would suggest actin reorganization. In general, the localization of GFP-actin in YTS cells is consistent with that of F-actin as demonstrated by phalloidin localization using total internal reflection fluorescence (TIRF) microscopy (Supplemental Figure 1; supplemental material available online with this article; doi:10.1172/JCI44862DS1). Using live-cell confocal microscopy, YTS GFP-actin cells counterstained with DRAQ5 to allow visualization of the nucleus were not observed to sequester the GFP signal preferentially into the cortex after addition of vehicle to the imaging chamber media. In contrast, the addition of IL-2 to the imaging chamber resulted in the rapid sequestration of the GFP into the cell cortical regions (Figure 1C). This effect of IL-2 was observed repeatedly, and a quantitative algorithm was applied to define the change in GFP intensity in the cortical region relative to that found in the total cell. When compared with the fluorescence intensity present in the cortical region at the time of addition of IL-2 to the media or in vehicle-treated cells, this value reproducibly increased over time in IL-2-exposed cells (Figure 1D). This margination of fluorescence into the cell cortex suggests that IL-2 can increase NK cell cortical F-actin. To provide independent biochemical confirmation, F-actin containing fractions of YTS cells expressing GFP-actin was prepared by centrifugation using speeds that leave monomeric actin in solution. Using this approach, IL-2 stimulation resulted in increased GFP-actin or endogenous actin in the precipitate (Figure 1E). This finding was specific, as the amount of histone $\mathrm{H} 1$ present in the fractions did not increase in those prepared from IL-2-stimulated cells, demonstrating an effect of IL-2 upon actin filamentation in NK cells.

Effect of IL-2 on NK cell F-actin reorganization and function is WASP independent. The simplest explanation for how IL-2 could be functioning to increase F-actin content in NK cells would be via an increase in WASp levels. To evaluate this possibility, YTS or ex vivo NK cells were treated with IL-2 and total cellular WASp levels evaluated by Western blot analysis. After IL-2 incubation, neither cell culture demonstrated appreciable increases in WASp levels (Supplemental Figure 2, A and B). Alternatively, IL-2 might further induce function from existing WASp. Even though the phenomenon of IL-2-induced restoration of F-actin accumulation at the lytic synapse was originally observed in WAS patient cells harboring an aberrant WASP gene (17), IL-2 could still be acting by enabling additional functional capacity from mutant WASp. In consideration of true WASp independence of IL-2, we were able to utilize ex vivo NK cells from a patient with WAS due to a complete deletion of exons 1-12 of the WASP gene and no detectable WASp (22). Although ex vivo NK cells from this patient could form contacts with target cells, they had a reduced ability to accumulate F-actin at the IS compared with that of ex vivo NK cells from a control donor (Figure 2A and Supplemental Figure 3). When the patient ex vivo NK cells were activated with IL-2, however, increased F-actin was found at the IS. This was quantified across multiple cells using a 
A
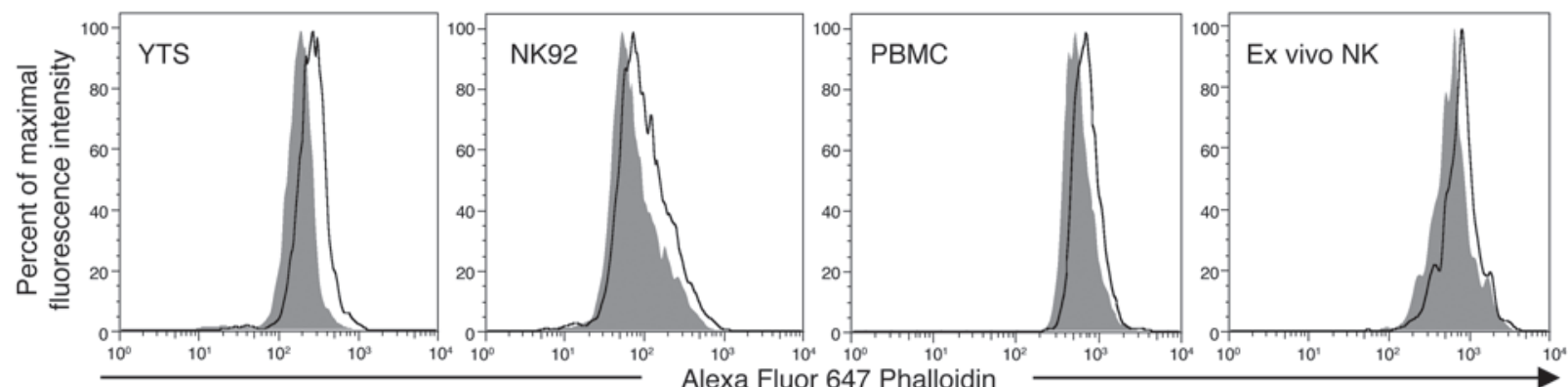

B
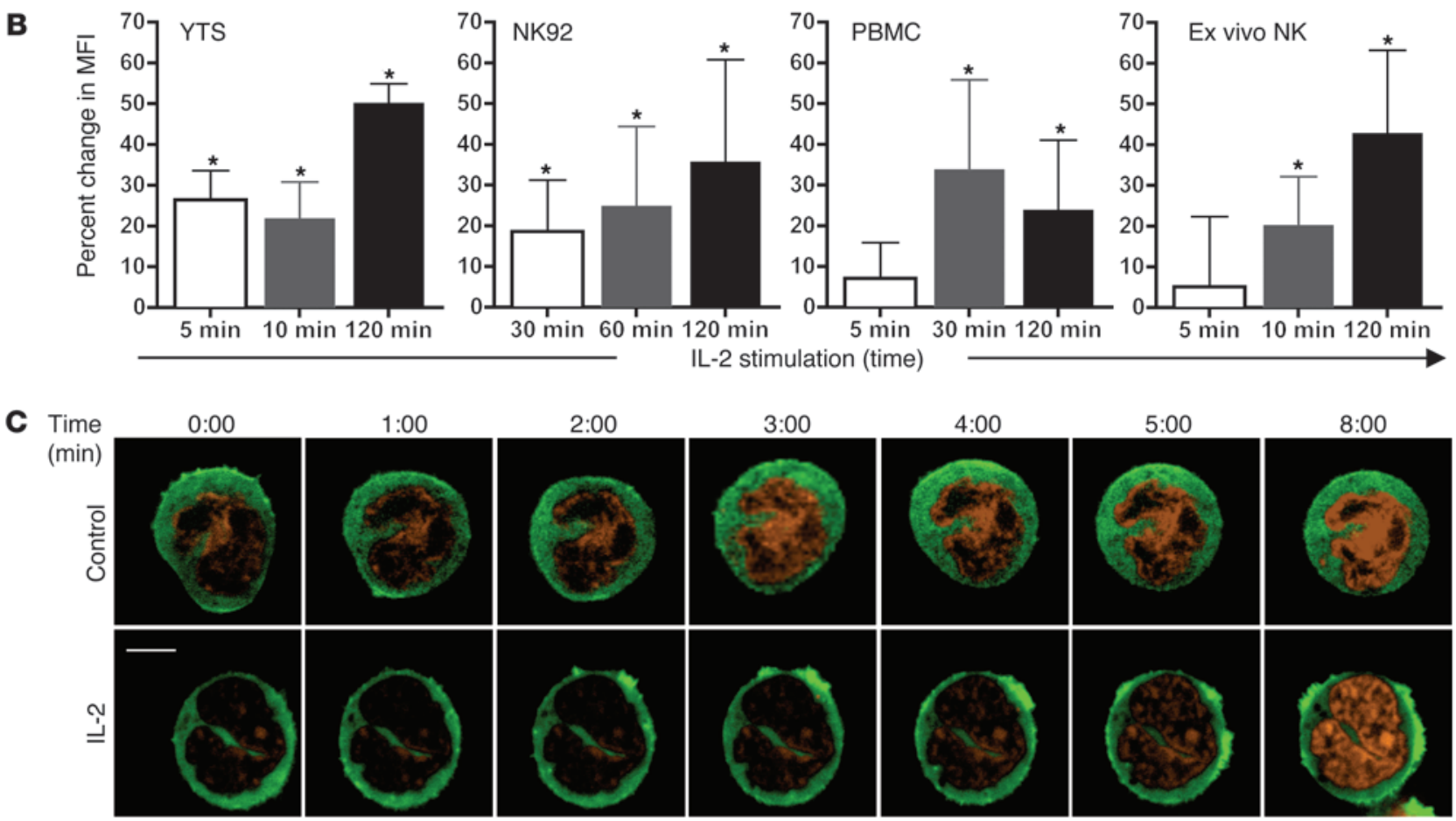

D

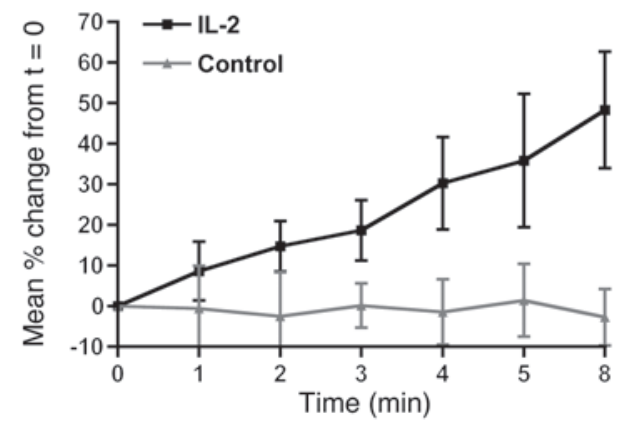

$\mathbf{E}$

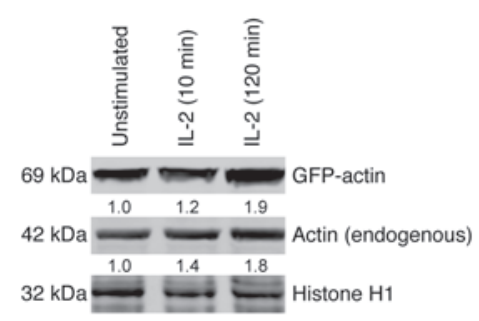

Figure 1

IL-2 promotes intracellular F-actin content in NK cells. (A) YTS, NK92, PBMC, or ex vivo NK cells were treated with $125 \mathrm{U} / \mathrm{ml}$ of IL-2 for 30 minutes, fixed, permeabilized, and stained with phalloidin Alexa Fluor 647. Percentage of maximal fluorescence intensity of Alexa Fluor 647 is demonstrated for control (gray) and IL-2-treated (white) cells. In PBMCs, only the CD56 ${ }^{+}$CD $3-$ cells were selected. (B) Phalloidin MFI induced by IL-2 over that in control-treated cells according to time of in vitro IL-2 or vehicle exposure (error bars demonstrate SD; ${ }^{*}<<0.05$ compared with control-treated cells). (C) Distribution of actin-GFP in control-treated (top) or IL-2-treated (bottom) YTS GFP-actin cells using real-time confocal microscopy. GFP-actin fluorescence is depicted in green, and DRAQ5 DNA dye (nuclear material) is depicted in red. Time $=0: 00$ represents the addition of IL-2 or vehicle to the imaging chamber. Scale bar: $5 \mu \mathrm{m}$. (D) Calculated change from time $=0: 00$ in mean area intensity of peripheral GFP-actin over the first 8 minutes in control-treated cells (gray triangles) compared with IL-2-treated cells (black squares). Each point represents the mean \pm SD from 10 cells, and the IL-2 and control data sets are significantly different $(P=0.0023)$ via a 2-tailed Mann-Whitney $U$ test. (E) Insoluble F-actin-rich cell fractions prepared from unstimulated, 10-minute, or 120-minute IL-2-stimulated YTS GFP-actin cells were evaluated by Western blot analysis for GFP-actin (top), endogenous actin (middle), and histone H1 (bottom). The numbers beneath the blots provide the densitometric ratio of the GFP or actin signal to that present in the unstimulated lane and are representative of 2 repeats. 
A
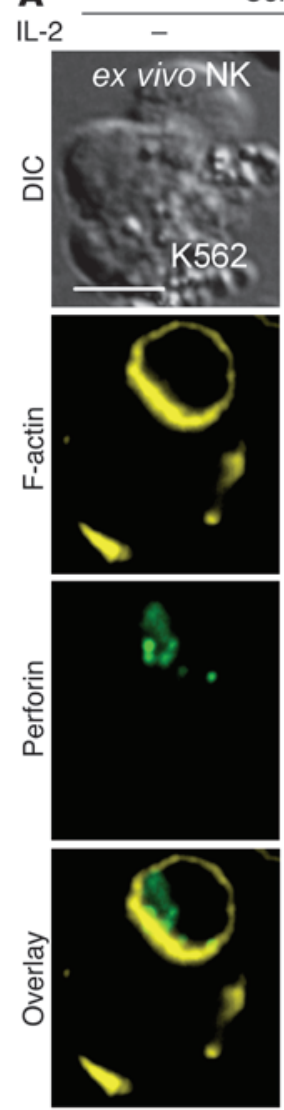

Control
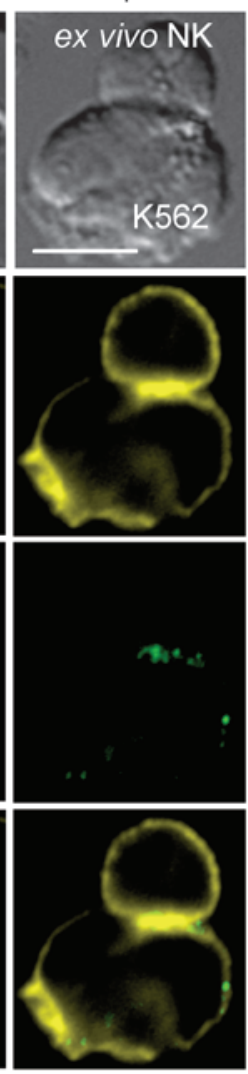

WASPdel
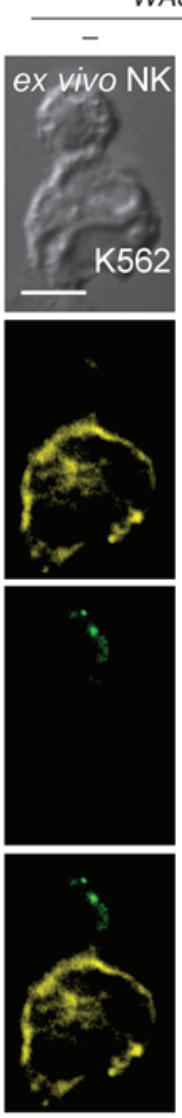
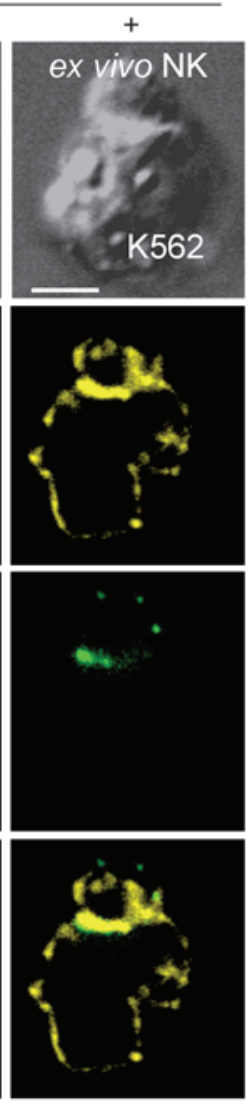

B

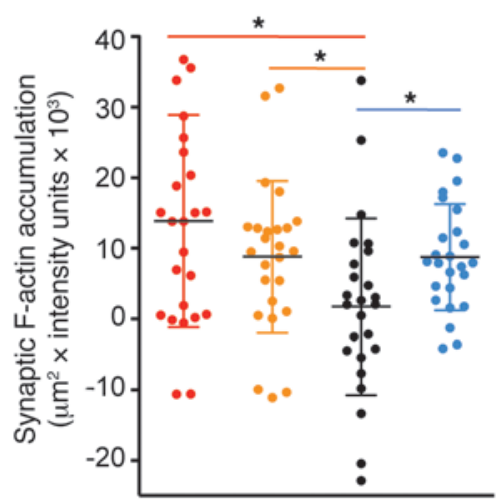

- Control ex vivo NK+K562

- IL-2 control ex vivo NK+K562

- WAS ex vivo NK+K562

- IL-2 WAS ex vivo NK+K562

C

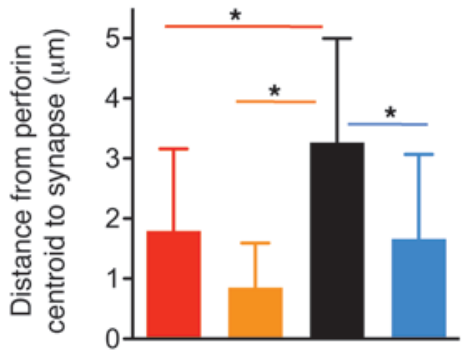

\section{Figure 2}

IL-2 restores F-actin accumulation at and lytic granule polarization to the NK cell IS. (A) Representative examples of the NK cell IS formed between resting or 30-minute IL-2-stimulated ex vivo NK cells obtained from either a control donor (control) or a WAS patient with a complete deletion of the WASP gene (WASPdel) with K562 target cells (additional examples in Supplemental Figure 3). Images show DIC (top panel) and confocal fluorescence microscopy for F-actin using phalloidin (yellow) and perforin (green) as well as an overlay of fluorescent channels. Scale bars: 5 um. (B) Quantification of F-actin accumulation at the IS formed between K562 target cells and ex vivo NK cells of control (red), IL-2-activated control (orange), WASPdel patient (black), and IL-2-activated WASPdel patient (blue). Each spot represents the calculated F-actin accumulation value (see Methods for details) from a single cell; the horizontal bar represents the mean of all cells shown and the vertical bars \pm SD. Significant differences among group means are shown. ${ }^{\star} P<0.05 ; n \geq 25$. (C) Measurement of lytic granule polarization as the shortest distance from the centroid of the entire lytic granule region to the IS in each of the ex vivo NK cell-target cell combinations. Bar colors correspond to those used in B and display mean + SD. Mean distance in resting WASPdel patient ex vivo NK cells was different from that in the other cells measured. ${ }^{*} P<0.05 ; n \geq 25$.

previously validated algorithm (23). In unstimulated patient ex vivo NK cells, there was no mean synaptic F-actin accumulation, but IL-2 induced levels to those found in control cells (Figure 2B). This increase in F-actin content was also associated with maturation of the IS, as only after IL-2 stimulation was perforin able to polarize to the IS in patient ex vivo NK cells (Figure 2A), as measured by the distance of the perforin region centroid to the synapse across multiple cells (Figure 2C). Thus, even in the absence of the WASP gene, IL-2 is able to promote actin reorganization.

As an alternative to the WASP deleted cells, the small molecule inhibitor of WASp, wiskostatin, was utilized. Wiskostatin stabilizes the autoinhibited conformation of WASp, thus blocking its ability to promote actin branching (24). Wiskostatin also inhibits N-WASp, which is a ubiquitously expressed WASp homolog. While N-WASp is not able to compensate for WASp in patients with WAS, it is possible that IL-2 was inducing function in NK cells through N-WASp. In NK92 or YTS cells or in
PBMCs, wiskostatin pretreatment did not reduce WASp levels (Supplemental Figure 2, A and B), but was able to inhibit the majority of cytolytic activity (Figure 3A). Addition of IL-2 to the cytotoxicity assay, however, improved function in both control and wiskostatin-treated NK92 cells or PBMCs. Control-treated YTS cells had only minimal increase in cytotoxicity after IL-2 stimulation, but IL-2 restored activity in wiskostatintreated cells to control levels. To determine whether IL-2 could induce a target cell-independent effect in the presence of wiskostatin, inhibitor- or vehicle-treated NK92 cells, YTS cells, or PBMCs were treated with IL-2 and evaluated for F-actin content. Although wiskostatin pretreatment reduced F-actin levels (at least in NK-92 cells and PBMCs, presumably by preventing new F-actin formation during actin turnover), addition of IL-2 to wiskostatin-treated cells increased the F-actin content toward control levels (Figure 3B). Since WASp promotes actin branching, we also evaluated the ultrastructure of F-actin at the IS in 
A
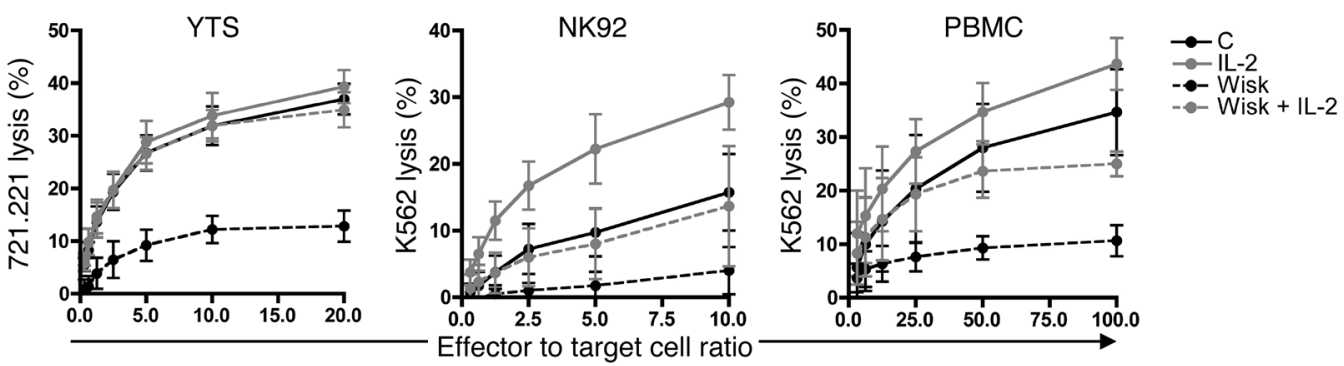

B
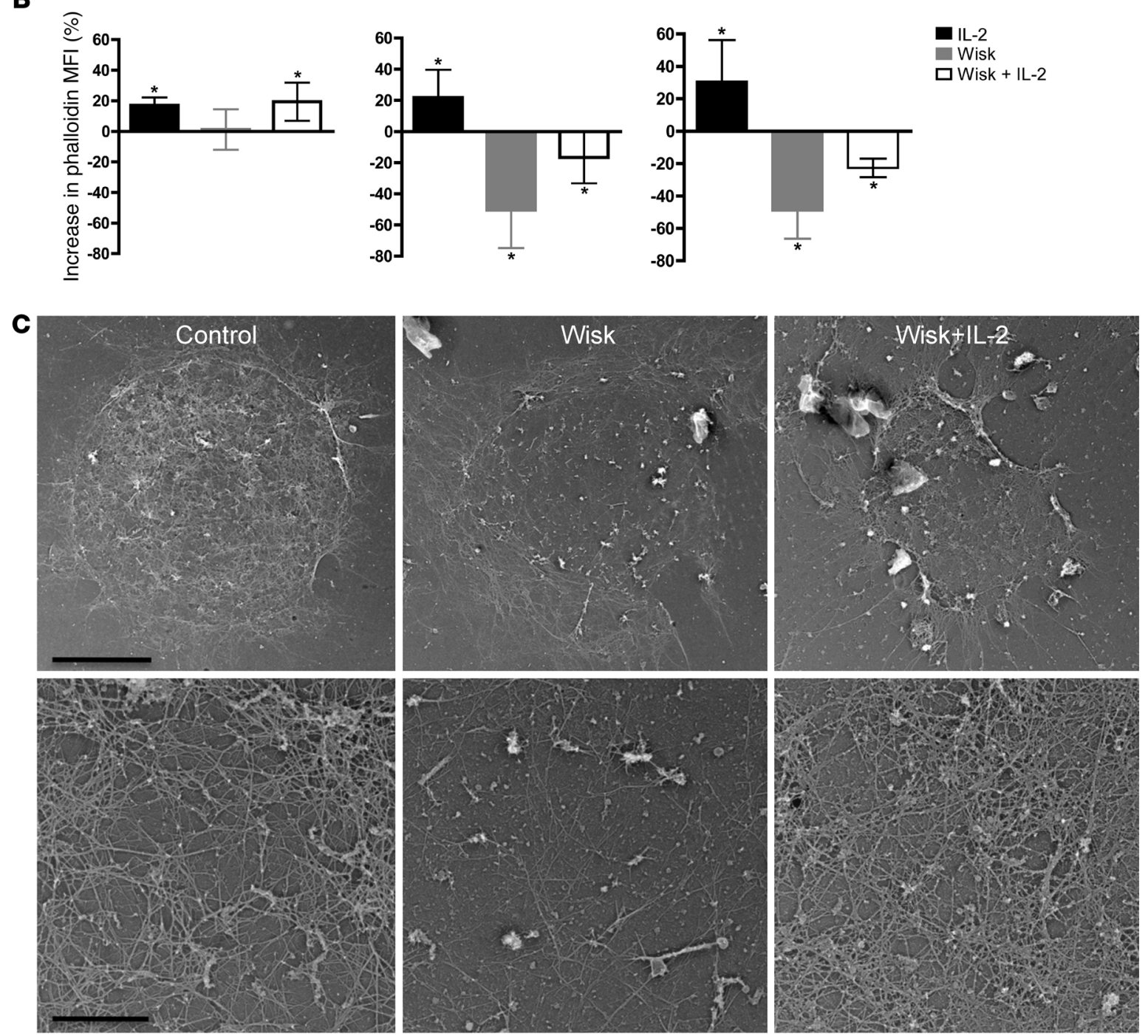

\section{Figure 3}

IL-2 increases cytotoxicity and F-actin reorganization in wiskostatin-treated NK cells. (A) Cytotoxic activity of YTS cells (left), NK92 cells (middle), or PBMCs (right) pretreated with vehicle control (c, black solid line), IL-2 (gray solid line), wiskostatin (Wisk, black dashed line), and wiskostatin followed by IL-2 (Wisk+IL-2, gray dashed line). Cells were incubated with IL-2 where specified for 30 minutes prior to the assay. K562 target cells were used for NK92, and 721.221 target cells for YTS and PBMCs. Cytotoxicity was measured by 4-hour ${ }^{51} \mathrm{Cr}$-release assays. Each point represents the mean of 5 individual assays, each performed in triplicate + SD. For each graph, decreases mediated by wiskostatin and increases in wiskostatin-treated cells mediated by IL-2 were significant ( ${ }^{*}<<0.05,2$-tailed Mann-Whitney $U$ test). (B) Change in F-actin content measured as percentage increase in phalloidin MFI via flow cytometry in YTS cells (left), NK92 cells (middle), or PBMCs (right). Vehicle control-treated cells were compared with IL-2-treated (black), wiskostatin-treated (gray), and wiskostatin- and IL-2-treated (white). Each bar represents the mean of 3 or more experiments $\pm S D\left({ }^{*} P<0.05\right.$, significant changes from control-treated cells). 30 -minute wiskostatin pretreatment was followed by 30-minute IL-2 treatment. (C) Representative TEM images of platinum rotary-shadowed, sonicated, and extracted YTS cells that had been adhered to glass coated with CD18 and anti-CD28. Prior to adherence to the glass, cells were treated with vehicle (left), wiskostatin (center), or wiskostatin followed by IL-2 (right). Cortical F-actin network detail is shown. Original magnification, $\times 6000$ (scale bar: $5 \mu \mathrm{m}$ [top]) and $\times 30,000$ (scale bar: $1 \mu \mathrm{m}$ [bottom]). 
A
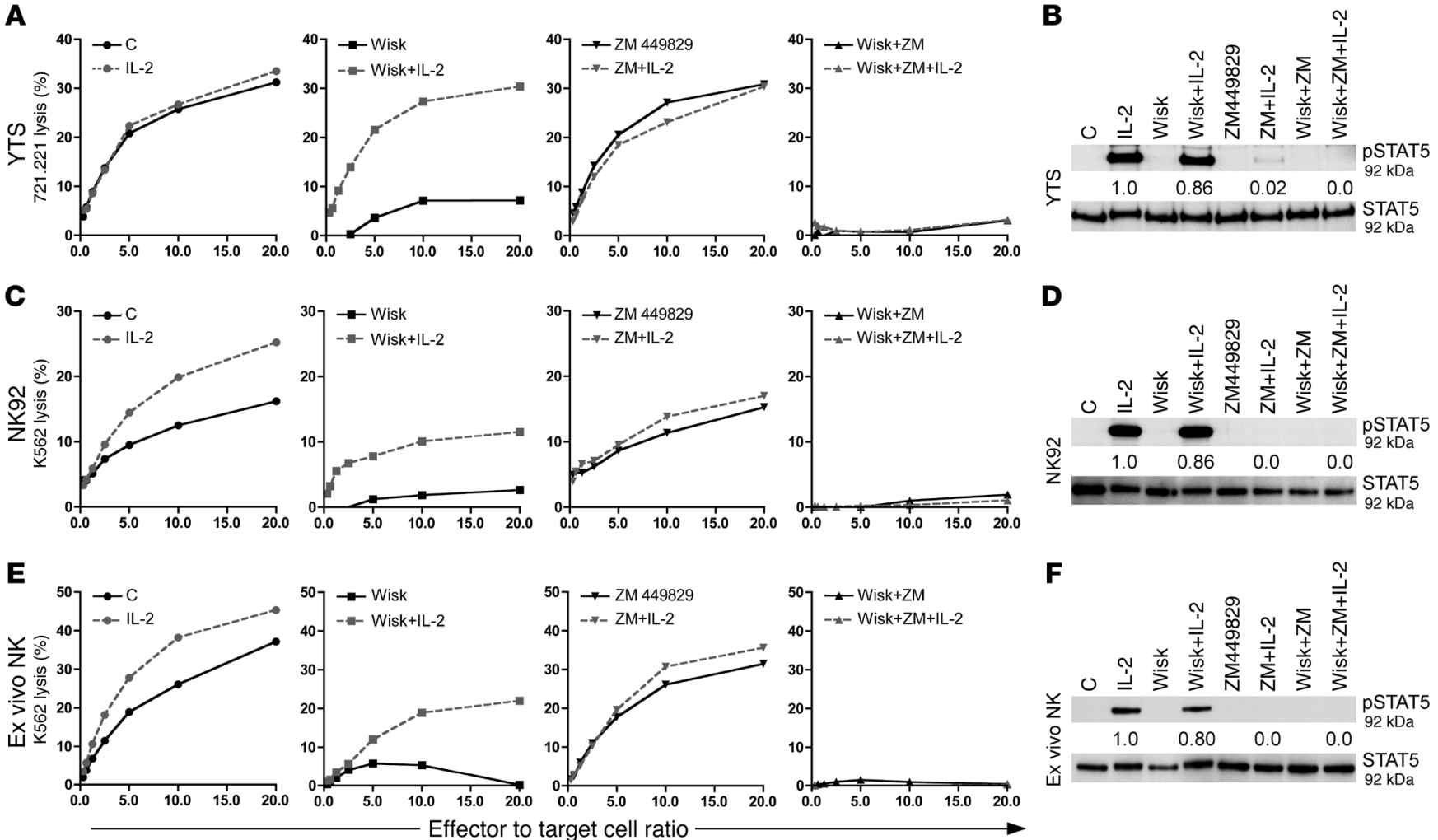

Figure 4

Requirement for IL-2 signaling to rescue NK cell cytotoxicity from WASp inhibition. YTS (A and B), NK92 (C and D), and ex vivo NK (E and F) cells were control (C, circles), wiskostatin (Wisk, squares), ZM 449829 (downward triangles), or wiskostatin and ZM 449829 (Wisk+ZM, upward triangles) treated prior to addition of media (solid black line) or IL-2 (dashed gray line) for 30 minutes. Each column of graphs depicts the effect of IL-2 (dashed line) added to the respective control or inhibitor-treated cells (solid line). The control cytotoxicity for all of the treatments is in the left-most graph (black circles). Cytotoxic activity of treated cells was measured against 721 or $\mathrm{K} 562$ target cells in 4 -hour ${ }^{51} \mathrm{Cr}$-release assays. Individual points represent the mean of triplicates, and individual experiments are representative of $2-5$ repeats. (B, D, F) A portion of the cells prepared for cytotoxicity was used to prepare whole-cell lysates, which were evaluated for the presence of phosphorylated (top) and total (bottom) STAT5 by Western blot analysis. Total STAT5 was detected using the same membrane used for phosphorylated STAT5 after stripping and reprobing. Numbers beneath each lane represent densitometric ratios for pSTAT5 to total STAT5.

activated NK cells using platinum replica electron microscopy. YTS cells were adhered to glass coated with anti-CD18 and antiCD28 to promote adhesion and activation and then extracted to remove all but the cell cortex. In control-treated activated cells, a branched actin network was evident, which was diminished when cells were treated with wiskostatin prior to activation (Figure 3C). When the wiskostatin-pretreated cells were treated with IL-2 during activation, however, a branched actin network was restored, albeit perhaps qualitatively distinct from control-treated cells. Together, these results suggest that IL-2 can act directly upon NK cells to circumvent WASp and N-WASp to promote NK cell cytotoxicity and F-actin organization.

We next wanted to determine whether the WASp-independent effect of IL-2 in promoting NK cell function was dependent upon classical IL-2 signaling. Thus, we utilized the JAK3 inhibitor ZM449829 (25) to interfere with IL-2 signal transduction. While YTS, NK92, or ex vivo NK cells pretreated for 30 minutes with ZM449829 were not inhibited in cytotoxicity, IL-2 was no longer able to rescue cytotoxicity after wiskostatin pretreatment (Figure 4, A, C, and E). To define the IL-2 signal in the presence of ZM449829 and/or wiskostatin, STAT5 phosphorylation was evaluated by
Western blot analysis. IL-2 treatment of YTS, NK92, or ex vivo NK cells robustly induced STAT5 phosphorylation. Wiskostatin pretreatment did not interfere, but ZM449829 blocked IL-2-induced STAT5 phosphorylation (Figure 4, B, D, and F). Thus, while wiskostatin does not appear to interfere with IL-2 signal generation, IL-2 signaling was required for the ability of IL-2 to circumvent inhibition of WASp in NK cells.

In vivo impact of IL-2 upon NK cells in WAS. Since WAS is associated with a potentially clinically relevant deficiency of NK cell function, we hypothesized that in vivo IL-2 administration to patients might induce the WASp-independent NK cell effects observed in vitro. Therefore, a clinical trial of IL-2 administration in WAS was organized, and a patient with a $134 \mathrm{C}>\mathrm{T}$ WASP mutation was treated with $0.5 \times 10^{6} \mathrm{U}$ of IL- $2 / \mathrm{m}^{2}$ subcutaneously for 5 days. Treatment was repeated twice at 2 -month intervals (Figure $5 \mathrm{~A}$ ). The patient tolerated IL-2 injections and experienced local reactions (Figure $5 \mathrm{~B}$ ), but otherwise finished the protocol uneventfully. A variety of assessments were performed in consideration of the impact of in vivo IL-2 administration, including NK cell cytotoxicity against K562 target cells measured among ex vivo PBMCs. As expected, the patient had decreased NK cell cytotoxicity pretreatment compared 
A

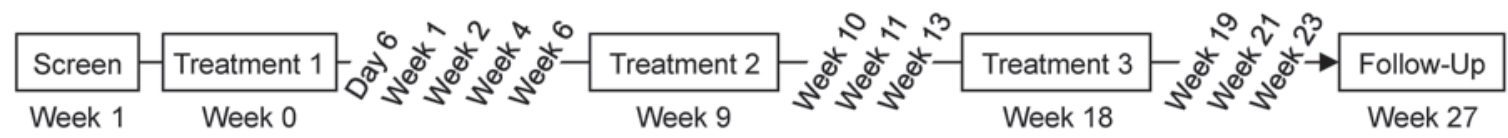

B
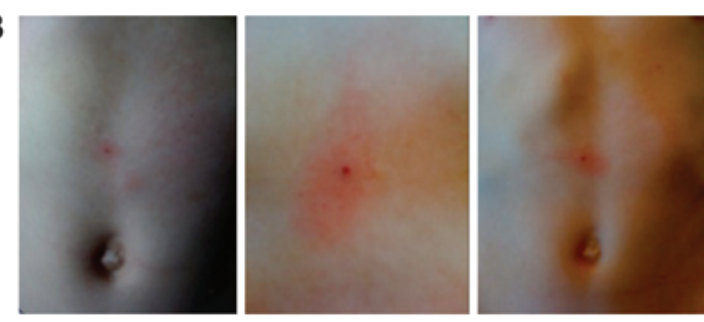

D

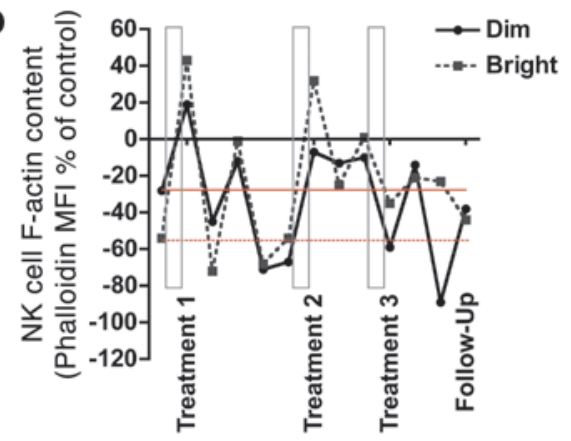

C $257 \rightarrow-$ Control
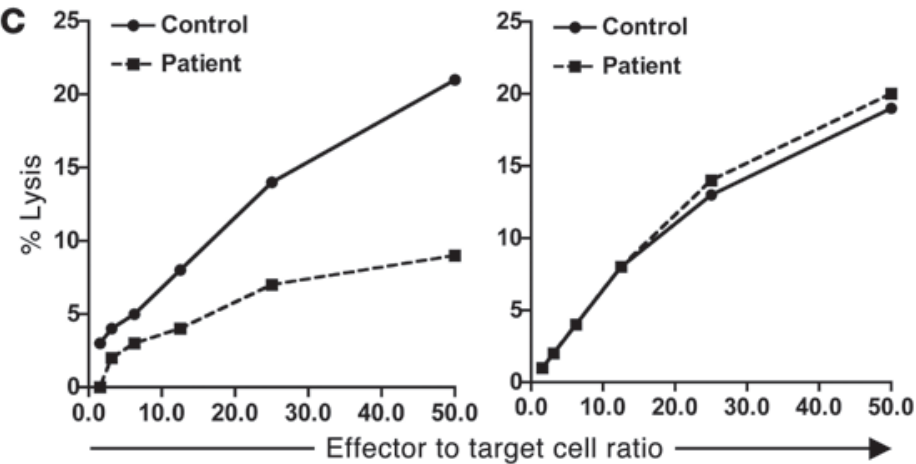

Figure 5

In vivo IL-2 administration enhances NK cell cytotoxicity and F-actin content in a WAS patient ex vivo. A patient with grade 2 WAS was enrolled and treated with subcutaneous IL-2 using $0.5 \times 10^{6} \mathrm{U} / \mathrm{m}^{2} / \mathrm{d} \times 5 \mathrm{~d}$ for each of 3 treatments as listed (A). Blood was drawn immediately prior to each treatment cycle as well as at the time points specified. (B) Examples of the local reactions noted at the IL-2 injection site. (C) NK cell cytotoxicity using patient PBMCs (dashed line) in ${ }^{51} \mathrm{Cr}$-release assay against $\mathrm{K} 562$ target cells from the initial screen (week -1) and the penultimate blood draw (week 23) compared with PBMC of an individual healthy donor (not treated with IL-2, solid line). (D) F-actin content in patient CD56 dim (solid line) and CD56bright (dashed line) NK cells expressed as the MFI percentage of that identified in control over the course of the treatment protocol. The red and orange lines represent the CD56 dim and CD56 bright pretreatment values, respectively, and the open rectangles correspond to the 5-day periods of IL-2 administration.

with control, with values similar to other patients having analogous WASP mutations $(16,17)$. After IL-2 administration (week 23), however, cytotoxicity was similar to control (Figure 5C). NK cell cytotoxicity was measured in samples obtained throughout the protocol and expressed as lytic units ( $\mathrm{LU}_{20}$, lytic units at $20 \%$ lysis) per NK cell. While there was not an increase in response immediately following all 3 courses of IL-2, after the second and third, the baseline $\mathrm{LU}_{20}$ remained elevated above the level present prior to starting the protocol (Supplemental Figure 4A). This suggests some lasting impact of the IL-2, even though pharmacokinetic assessments of similar doses in other populations would predict that circulating administered IL-2 would no longer be apparent (26). The elevated baseline lytic units at the end of the protocol were not reflected by shifts in the NK cell population, as the percentage of NK cells or distribution of CD56 $6^{\mathrm{dim}}$ and $\mathrm{CD} 56^{\text {bright }} \mathrm{NK}$ cells was not consistently altered over the treatment period (Supplemental Figure 4, B and C). There were also no major consistent shifts in the percentages of total $\mathrm{CD} 3^{+} \mathrm{T}$ cells, although individual subpopulations were not evaluated (Supplemental Figure 4D).

To evaluate the effect of in vivo IL-2 upon NK cell F-actin content, ex vivo patient cells were studied using fluorescent phalloidin. WAS patient CD56 ${ }^{\mathrm{dim}}$ or CD56 $6^{\text {bright }} \mathrm{NK}$ cells had lower pretreatment phalloidin MFI compared with control (Figure $5 \mathrm{D})$. After the first and second courses of IL-2 administration, increases in F-actin content in both CD $56^{\mathrm{dim}}$ and CD $56^{\text {bright }} \mathrm{NK}$ cells were observed relative to control. There was an accentuated effect in the CD56 bright population, which may be a feature of high-affinity IL-2 receptor, which is preferentially expressed on this subpopulation (27). To directly measure F-actin in NK cells from the patient in a functionally relevant context, the lytic IS formed with K562 target cells ex vivo was evaluated. Accumulation of F-actin and polarization of perforin were identified at the IS after 30 minutes in control donor, but not pretreatment WAS patient, cells (Figure 6, A and B). Patient samples analyzed after each of the 3 courses of IL-2, however, demonstrated a restored lytic synapse with F-actin accumulation and perforin polarization (Figure 6, A-C). In all 3 posttreatment patient samples, synaptic F-actin content or perforin polarization was significantly improved compared with pretreatment and was similar to control donor values. These data demonstrate a functional impact of IL-2 on the NK cell population in vivo in a patient with WAS and further suggest the access of IL-2 to a WASp-independent pathway for NK cell function.

WASp-independent activity of IL-2 requires WAVE2. We next wanted to determine how IL-2 was accessing NK cell actin reorganization and function independently of WASp. Although NK cells rely heavily upon WASp as an Arp2/3 activator for F-actin branching, the major protein serving this function in T cells is WAVE2 (1). 
A

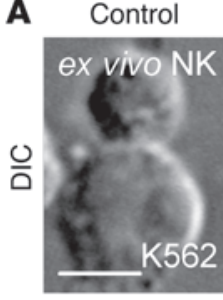

WAS pre
ex vivo NK
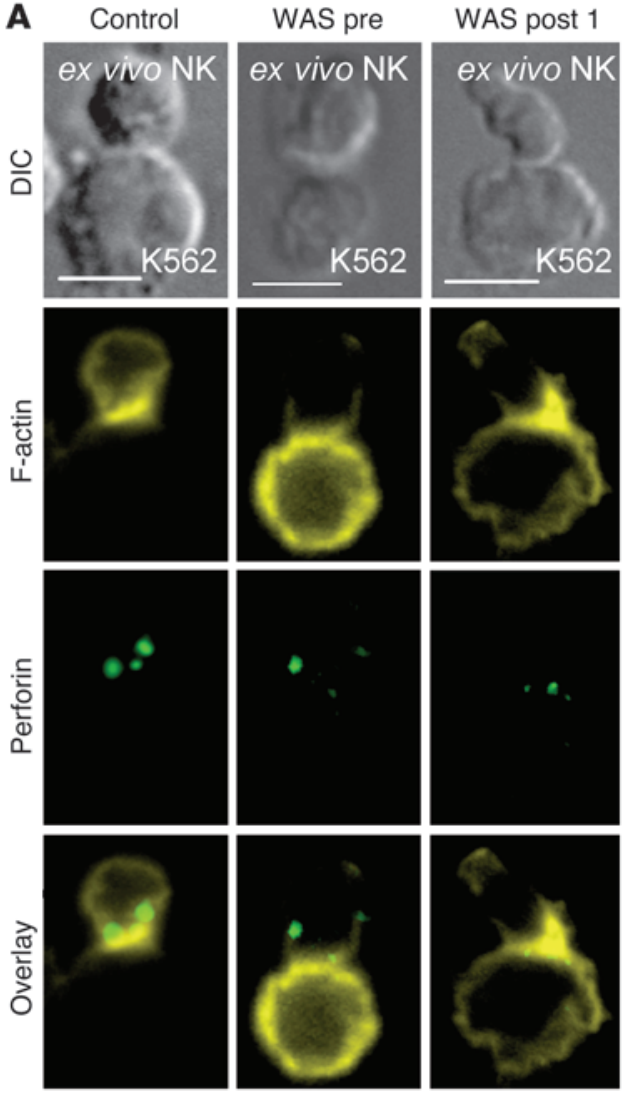
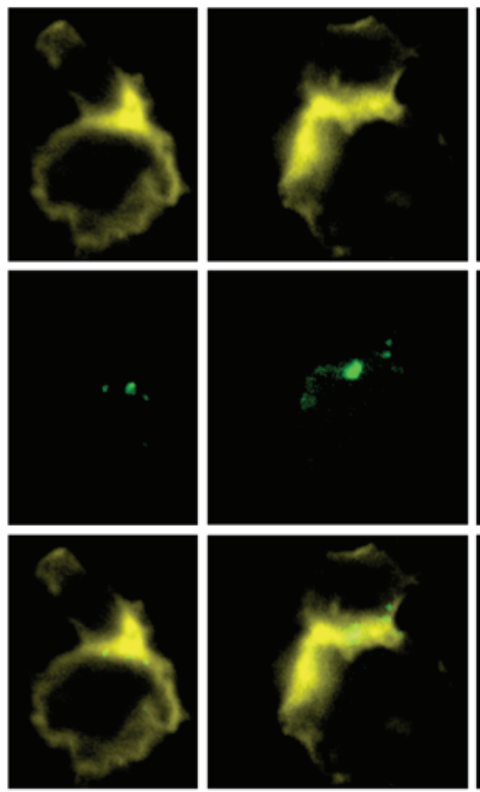

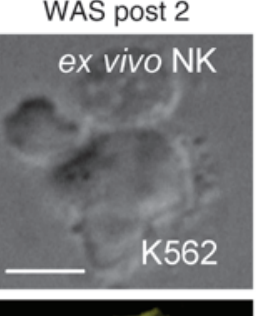

WAS post 3
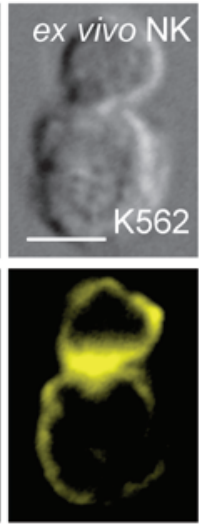
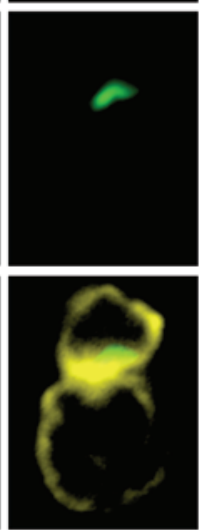

B

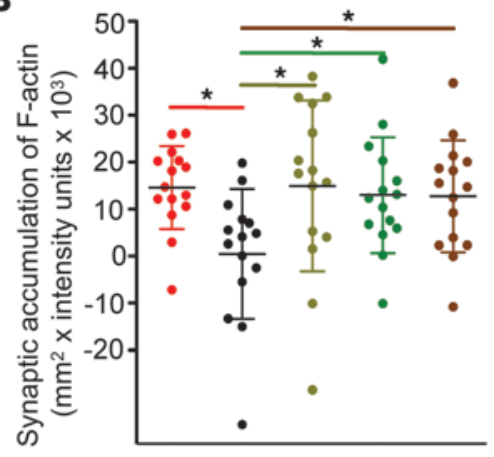

- Control ex vivo NK+K562

- WAS ex vivo NK+K562 Pretreatment

- WAS ex vivo NK+K562 Post-treatment 1

- WAS ex vivo NK+K562 Post-treatment 2

- WAS ex vivo NK+K562 Post-treatment 3

C

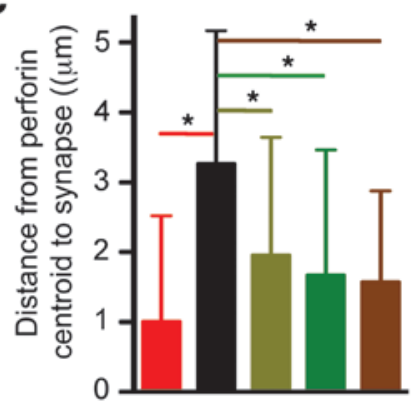

Figure 6

In vivo IL-2 administration of IL-2 to a WAS patient restores F-actin accumulation at and lytic granule polarization to the lytic IS in NK cells. (A) Representative images (from left to right) of the NK cell IS formed between an ex vivo NK cell from a control donor or the WAS patient prior to or after each of the 3 courses of IL-2 treatment and K562 target cell. A DIC image (top panel) and confocal fluorescence micrographs (bottom panels) depicting F-actin (phalloidin, yellow) and perforin (green) as well as an overlay of fluorescent channels. Scale bars: 5 um. Quantitation of F-actin accumulation at (B) and polarization of the lytic granule region to the IS (C) in control donor (red) and patient before (black) as well as after the first (light green), second (dark green), and third (brown) IL-2 treatments as presented in Figure 2 . Each mean represents 15 synapses $\pm \mathrm{SD}$ and significant differences are noted. ${ }^{*} P<0.05$.

Thus, we attempted to identify WAVE2 in NK cells and to determine whether levels were induced by IL-2 stimulation. WAVE2 was present in both YTS and ex vivo NK cells, but levels were not increased after 30 minutes of IL-2 stimulation (Figure 7, A and B). To determine whether WASp inhibition might uncover an ability of WAVE2 to be upregulated by IL-2, cells were pretreated with wiskostatin prior to stimulation. While wiskostatin pretreatment did not alter WAVE2 levels, they were not further increased by IL-2.

It was still possible, however, that IL-2 was activating WAVE2 in NK cells. WAVE2 activation requires Rac activation, acidic phospholipids, and WAVE2 serine phosphorylation (28). To determine whether the signal for WAVE2 activation was induced in NK cells after IL-2 stimulation, we evaluated the presence of active Rac using a GTP-Rac pull-down assay. GTP-Rac was increased more than 3 -fold after 30 minutes of IL-2 exposure and was not negatively affected by preincubation with wiskostatin (Supplemental Figure 5). This suggests that WAVE2 activation can occur after IL-2 stimulation and independently of WASp function. Because WAVE2 is phosphorylated upon activation (29) and specific serine phosphorylation is required for WAVE2 function (28), we next evaluated WAVE2 for serine phosphorylation following IL-2 stimulation in NK cells. YTS cells were pretreated with vehicle or wiskostatin and treated with IL-2 for 30 minutes; WAVE2 was isolated via immunoprecipitation. Phosphorylated STAT5 was measured in the supernatant to determine whether cells had received an IL-2 signal, and as expected, was only present after IL-2 exposure and was unaffected by wiskostatin (Figure 7C). Also as expected, WAVE2 was present in the immunoprecipitate (Figure 7D). IL-2 stimulation induced phosphoserine signal at the WAVE2 band (Figure 7, D and E). Although wiskostatin treatment decreased baseline phosphoserine levels at the WAVE2 band, they were induced approximately 8 -fold by IL-2 stimulation in wiskostatin-treated cells. This experiment was also performed using ex vivo NK cells. WAVE2 was present in the ex vivo NK cell immunoprecipitate, and IL-2 induced serine phosphorylation at the WAVE2 band (Figure 7, F and G). Similar to YTS cells, the baseline phosphoserine in the WAVE2 band was decreased by wiskostatin pretreatment, but was increased approximately 6-fold by IL-2. To confirm this finding in the absence of WASp, the WAVE2 immunoprecipitate from NK cells from the patient with a deleted WASP gene (Figure 2) was evaluated. IL-2 induced 
A

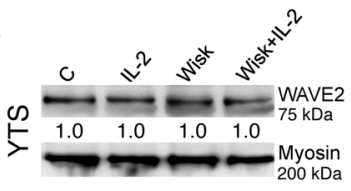

C

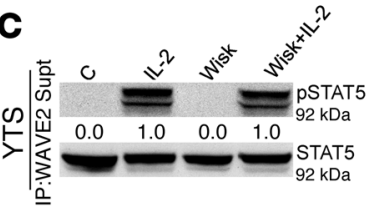

D

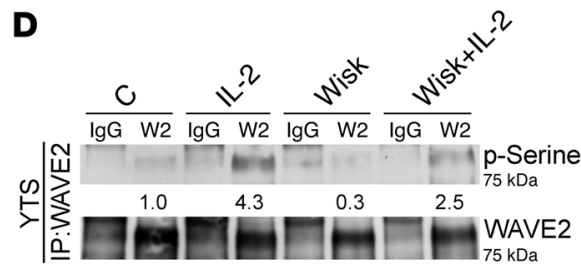

$\mathbf{F}$

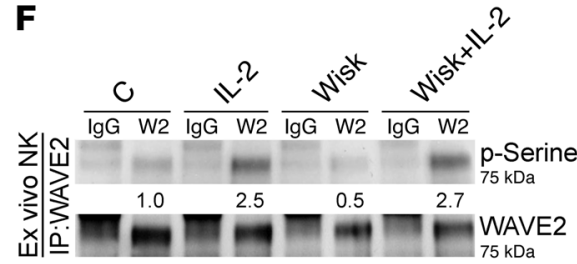

E

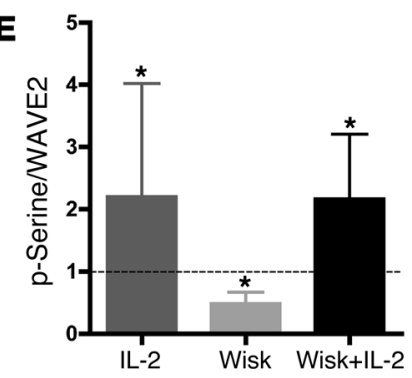

G

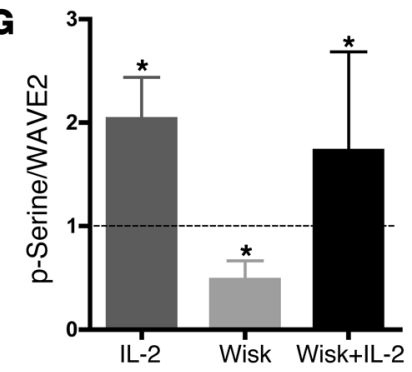

H

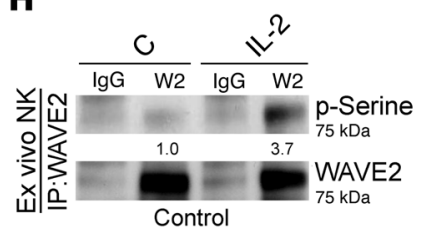

I

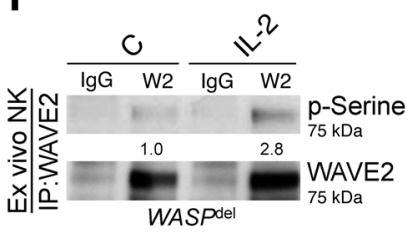

Figure 7

IL-2 stimulation of NK cells induces WASp-independent WAVE2 phosphorylation. YTS and ex vivo NK cells were pretreated with vehicle (C, control), wiskostatin (Wisk) and/or followed by IL-2 for 30 minutes. Whole-cell lysates of YTS (A) and ex vivo NK (B) cells were evaluated for WAVE2 by Western blot analysis. Membranes were stripped and reprobed for myosin-IIA as a loading control (bottom). Numbers beneath each lane represent densitometric ratios for WAVE2 protein normalized to loading control. (C) Supernatant of WAVE2 immunoprecipitate from YTS cells (treated as above) evaluated for the presence of phosphorylated and total STAT5. The WAVE2 immunoprecipitate from YTS (D and E) and ex vivo NK cells (F and $\mathbf{G}$ ) was evaluated first for phosphoserine (top) by Western blot analysis; then the membrane was stripped and reprobed for WAVE2 (bottom). $\lg \mathrm{G}$ immunoprecipitate $(\mathrm{IgG})$ is included as a control for the WAVE2 (W2) immunoprecipitate. Numbers beneath each lane represent densitometric ratios for phosphorylated WAVE2 protein relative to control normalized to total WAVE2 content. Western blot analyses are representative of 3 independent experiments from which the mean densitometric ratios are shown graphically (E and $\mathbf{G})$. Error bars show SD, and values significantly different from the mean in control-treated cells are noted $\left({ }^{*} P<0.05\right)$. WAVE2 or IgG immunoprecipitate from $(\mathbf{H})$ control, and (I) WASPdel patient NK cells (shown in Figure 2) that were pretreated with media control or IL-2 for 30 minutes was evaluated using Western blot analysis as for $\mathbf{D}$ and $\mathbf{F}$. phosphoserine levels at the WAVE2 band in both control and patient NK cells (Figure 7, H and I). Thus, IL-2 activated WAVE2 in NK cells as determined by phosphorylation, even in the presence of WASp inhibition or in the absence of WASp.

To determine whether WAVE2 was required for the WASpindependent effect of IL-2 upon NK cell function, we established WAVE2 siRNA in YTS and ex vivo NK cells. Compared with control siRNA, WAVE2-specific siRNA reduced WAVE2 levels by $70 \%-80 \%$ (Figure 8, A and B). Cells treated with siRNA were then further treated with wiskostatin and/or IL-2 and evaluated for cytotoxicity. In control siRNA-treated cells, wiskostatin inhibited killing and IL-2 was able to restore cytotoxicity to baseline levels (Figure 8, C and D). WAVE2 siRNA negligibly affected baseline cytotoxic activity, but completely inhibited the ability of IL-2 to restore cytotoxicity after wiskostatin treatment (Figure 8, C and D). Thus, WAVE2 was required for WASp-independent access to cytotoxicity. To determine whether this was reflected at the level of F-actin, the target cell-independent ability of IL-2 to increase F-actin content and partially compensate for WASp inhibition was evaluated. In control siRNA-treated cells, IL-2 increased F-actin content and IL-2 was still able to increase F-actin content in wiskostatin-treated cells (Figure 8, E and F). In WAVE2 siRNA-treated cells, however, the ability of IL-2 to increase F-actin content in wiskostatin-treated cells was lost. To confirm that IL-2 signaling was still active in these cells and had not been perturbed by reduced WAVE2 expression, STAT5 phosphoryla- tion levels were determined in YTS cells. In all cases, IL-2 was still able to robustly induce STAT5 phosphorylation (Figure 8G), demonstrating that the role of WAVE2 is downstream of the IL-2 signal. Thus, our results demonstrate the existence of a WASpindependent pathway for actin reorganization accessible by IL-2 and dependent upon WAVE2.

\section{Discussion}

NK cells depend upon actin reorganization to effectively form and access cytolytic function through the IS. A central role for WASp in this process has been defined through studies of ex vivo NK cells from patients with WAS $(11,15,17)$. Patient cells are defective in cytotoxicity and have a reduced ability to accumulate F-actin at the IS. Since patients with WAS have susceptibility to herpesviruses and hematologic malignancies, it is feasible that their deficiency of NK cell function is clinically relevant. The identification of deficient NK cell function in WAS presented a challenge, however, because IL-2 as a standard additive to human NK cell culture restores NK cell cytotoxicity. In the present work, we defined the mechanism of this IL-2-mediated effect and therein identified a second pathway for actin organization in NK cells. We also applied it to WAS to determine proof of principle in consideration of clinical application.

Unexpectedly, we found IL-2 had a direct impact upon F-actin in NK cells. When provided in solution, it increased F-actin content independently of the presence of target cells. Thus, IL-2 would be 

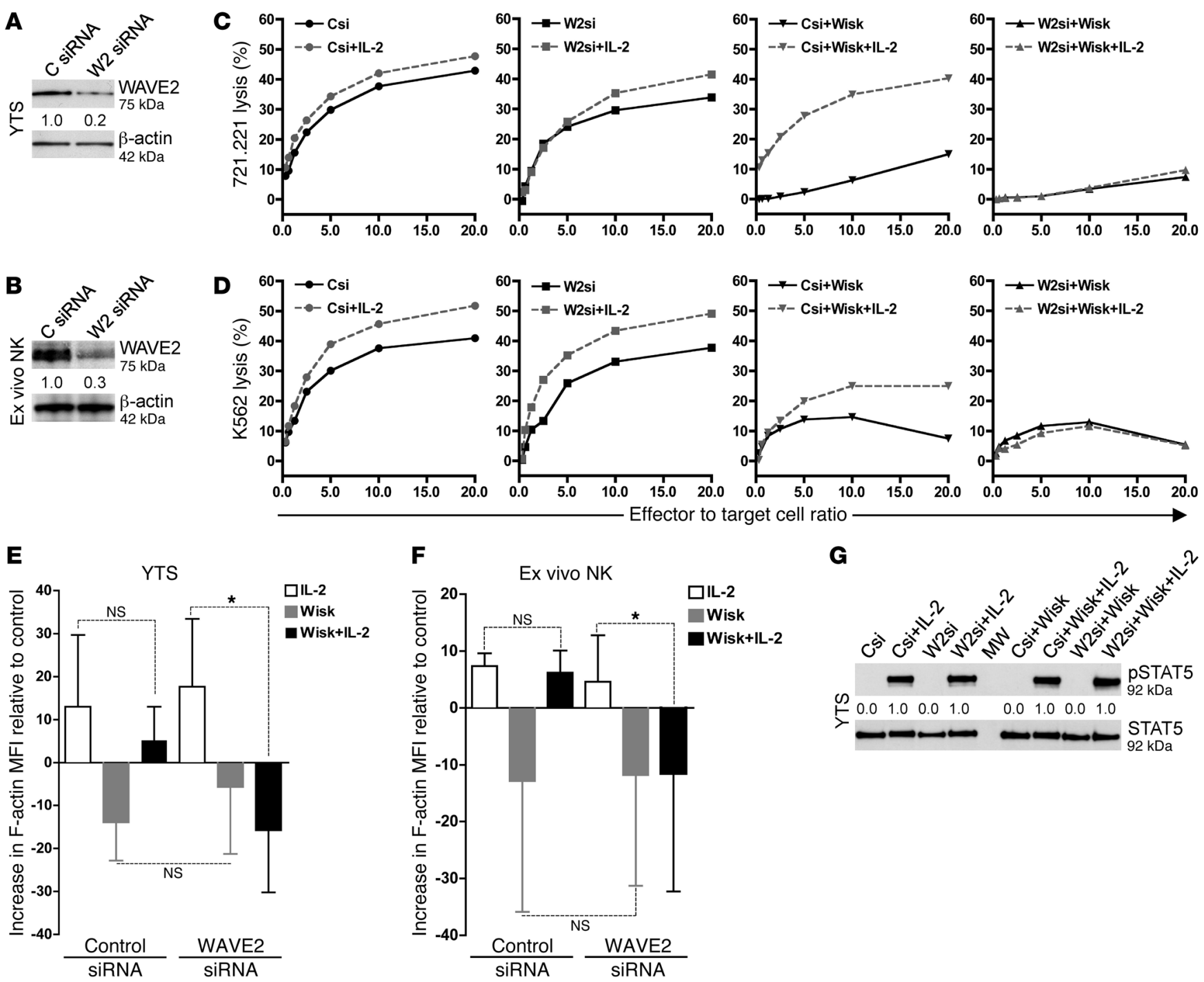

Figure 8

Requirement for WAVE2 in IL-2-induced rescue of WASp inhibition in NK cells. (A) YTS or (B) ex vivo NK cells nucleofected with control or WAVE2-specific siRNA for 24 hours were lysed and evaluated for the presence of WAVE2 (top) and $\beta$-actin expression by Western blot analysis. Nucleofected YTS (C, E, G) or ex vivo NK cells (D and F) were treated with wiskostatin or vehicle for 30 minutes followed by media or IL-2 for 30 minutes and then evaluated for cytotoxic activity against ${ }^{51} \mathrm{Cr}$-labeled (C) 721.221 or (D) K562 target cells in a 4-hour ${ }^{51} \mathrm{Cr}$-release assay, respectively. Cells were control siRNA (Csi, circles), WAVE2 siRNA (W2si, squares), control siRNA and wiskostatin (Csi+Wisk, downward triangles), or WAVE2 siRNA and wiskostatin (W2si+Wisk, upward triangles) treated prior to addition of media (solid black line) or IL-2 (dashed gray line) for 30 minutes. Each column of graphs depicts the effect of IL-2 (dashed line) in each condition. ( $\mathbf{E}$ and $\mathbf{F}$ ) F-actin content via phalloidin MFI in cells treated as per B and D, respectively, using flow cytometry (expressed as percentage change in MFI relative to unstimulated cells). Values represent means of 3 independent experiments and error bars show SD. Comparison of means is noted as not significantly or significantly $\left({ }^{*} P<0.05\right)$ different. (G) Phosphorylated and total STAT5 in YTS cells treated as per part B via Western blot analysis. Membranes were first probed for pSTAT5 and then stripped and reprobed for total STAT5. Numbers beneath each lane represent densitometric ratios of PSTAT5 normalized to total STAT5.

expected to promote F-actin reorganization diffusely in NK cells since it would derive signal from abundant cell-surface IL-2 receptors (27). In support, diffuse real-time reorganization after IL-2 exposure was identified in GFP-actin YTS cells (Figure 1). The effect of IL-2 upon F-actin in suspension culture NK cells did not require WASp, as IL-2 was able to circumvent blockade resulting from the WASp-inhibitor wiskostatin. This demonstrated that a cell contact-independent stimulus could induce a distinct means of promoting F-actin organization.
F-actin induction by IL-2 also could occur independently of WASp when a cell contact was present. This was demonstrated using wiskostatin and patient cells completely lacking the WASP gene by increases in, or relocalization of, phalloidin staining as well as the ultrastructure of actin filaments. NK cell cytotoxicity, which requires actin reorganization, followed suit. The effect of IL-2 upon F-actin was unlikely to be via the induction of the WASp homolog N-WASp, since wiskostatin was originally defined for its ability to inhibit N-WASp (24). A distinct Arp2/3 activator was therefore pursued. 
IL-2 directly induced phosphorylation of the Arp2/3 activator WAVE2. This could occur independently of stimulatory inputs derivative from the lytic IS, as it was identified in NK cells in suspension (Figure 7). Although the regulation of WAVE2 is the subject of ongoing investigation, phosphorylation of the protein is required for WAVE2-mediated actin nucleation (28). Known signals inducing WAVE2 phosphorylation include serum starvation (28, 29), PDGF (29), and EGF (28), but not IL-2. In these systems, acidic phospholipids and Rac activation are also required for WAVE2 activation. In this light, we found Rac to be activated after IL-2 stimulation and to occur independently of WASp function (Supplemental Figure 5). The same was true for WAVE2 phosphorylation (Figure 7, D-I). Sufficient numbers of NK cells, however, were only available to perform these experiments using healthy donor cells treated with wiskostatin in vitro or cells from the WASp-deleted patient but not the patient treated with IL-2 in vivo. Regardless, since the WASp-independent restoration of cytotoxicity was dependent upon IL-2 signaling (Figure 3), it would appear that IL-2 has specific WASp-independent access to WAVE2 activation. Importantly, WAVE2 was required for the ability of IL-2 to rescue NK cells from WASp blockade, as demonstrated using siRNA. WAVE2, however, was not required for the majority of baseline cytolytic function in NK cells. Thus, IL-2 represents what we believe to be a novel WAVE2 activator and an alternative mechanism to that directed by WASp for accessing F-actin reorganization in NK cells.

The utility of WAVE2 suggests 2 distinct pathways to F-actin reorganization in NK cells. The first is dependent on WASp and presumably accessible through routine contact-dependent activation signals, such as those provided through lytic triggering receptors. This is likely to be the default pathway in NK cells, since they do not produce significant IL-2. This requirement is further suggested by the NK cell deficiency identified in WAS patients (16, 17). The second pathway uses WAVE2 and is inducible and accessible in NK cells through a soluble IL-2 signal. Once induced, however, this pathway can enable the directed accumulation of F-actin at the IS and likely permits WAVE2 to substitute for WASp at the IS downstream of activating receptors. It is presently unclear if the requirement for IL-2 is in targeting WAVE2 to the IS, or for activation of WAVE2 irrespective of subcellular localization. While a role for IL-2 has not been previously investigated, the WAVE2 pathway has been shown to be the default in T cells and required for function downstream of the TCR $(1,2,30,31)$. The role for WASp in $\mathrm{T}$ cells regarding TCR-induced actin accumulation is less clear (3, $4,32)$. It is possible, however, that some tonic exposure to IL-2 in the context of routine $\mathrm{T}$ cell expansion may constitutively enable the WAVE2 pathway in mature T cells. This pathway may be defective in WAS due to the requirement for WASp in production of IL-2 by human T cells. An IL-2 production defect in WAS patient $\mathrm{T}$ cells has been demonstrated after in vitro activation of cells using certain stimuli, including mitogens or immobilized anti-TCR antibodies (6-9) as well as some (19), but not all, (7) antigen-presenting cell systems. Decreased IL-2 production by WASp-deficient $\mathrm{T}$ cells after stimulation has also been defined in mice $(3,4,33)$.

This dichotomy establishes a difficult impasse in WAS, one in which NK cells can utilize neither the default WASp pathway for genetic reasons nor the WAVE2 pathway fully due to potentially inadequate IL-2 supply from T cells. Given that patients are affected by clinical conditions associated with defective NK cell defense, this suggests the opportunity to intervene by enabling the WASp-independent pathway therapeutically using IL-2. IL-2 had been previously administered with reported clinical benefit to a WAS patient who had intractable HSV infection, but detailed immune evaluations including those of NK cells were not performed (34). In the present work we were able to study the impact of IL-2 administration upon NK cells in a WAS patient. Although IL-2 therapy is not benign and has many associated adverse effects, the dose used in the patient treated was well tolerated, substantially lower than those typically associated with severe toxicities and similar to those in other studies not associated with severe toxicity (35). This may suggest an opportunity, however, to consider other potentially better tolerated therapeutic cytokines using the common $\gamma$-chain for signaling. In this light, we have preliminarily found that IL-15 can also induce actin content in NK cells and differences between the common $\gamma$-chain utilizing cytokines need to be considered in detail.

Our initial experience with in vivo IL-2 administration, however, demonstrated at least some degree of enhanced NK cell cytotoxicity, F-actin content, and lytic IS formation. While further study is indicated, these initial results also suggest some sustained impact of IL-2 on NK cells past what might be expected given the pharmacokinetics of IL-2 (26). This could potentially be explained by some restorative effect of IL-2 on patient T cells (as has been described in vitro; refs. 19, 36), perhaps enabling them to produce IL-2 and drive the WAVE2 pathway in NK cells, and represents a mechanism warranting future investigation. Importantly, however, as NK cell deficiency is present in even mild WAS patients (17) for whom hematopoietic stem cell therapies might not represent an acceptable risk, IL-2 may present a reasonable option. A majority of these more mildly affected patients live past childhood, but have a median event-free survival of 10 years with a $5 \%$ risk of lymphoid malignancy (18). Thus, intermittent application of IL-2 might facilitate NK cell surveillance functions to improve outcome in these more mildly affected patients and will be a focus of future work.

While the ability to access the WAVE2 pathway in NK cells therapeutically is potentially clinically relevant in WAS, it also provides insight into mechanisms underlying cooperation between adaptive and innate immunity. Specifically, T cells could provide IL-2 to enable enhanced WAVE2-dependent F-actin organization in NK cells upon their encountering a susceptible target. This defines a mechanism of how T cells may augment innate NK cell functions during an immune response. Since this can be accomplished with soluble IL-2, it could presumably be exploited by local or even distantly activated $\mathrm{T}$ cells. This presents a new potential mechanism for how adaptive immunity could cooperate with innate function in enhancing the response against host cells that may have become damaged, but do not present the specific antigen that would be recognized by responding $\mathrm{T}$ cells. Thus, dual pathways to actin reorganization can be differentially accessed and likely serve distinct functions in the immune response.

\section{Methods}

NK cells, cell lines, and in vitro treatments. The YTS and NK-92 immortalized NK cell lines, both derivative from malignant expansions of NK cells, as well as the target cell K562 erythroleukemia line and 721.221 lymphoblastoid line were grown and maintained in culture as described previously (37). PBMCs were isolated from healthy volunteers using Ficoll Hypaque (Amersham) from which NK cells were isolated by negative selection where specified using the human NK cell isolation kit-II (Miltenyi Biotec). Preparations of ex vivo NK cells were evaluated via flow cytometry to confirm purity, which was greater than $95 \% \mathrm{CD}^{2} 6^{+} \mathrm{CD}^{-}$with less than $1.0 \% \mathrm{CD}^{+}$ 
cells. PBMCs or ex vivo NK cells were used for experiments immediately after isolation. Splenocytes from a previously defined patient with WAS due to complete deletion of exons 1-12 of WASp (22) were used from cryopreserved samples where indicated for preparation of NK cells.

Where specified, cells were treated in vitro with $3 \mu \mathrm{M}$ wiskostatin (CalBiochem) or $1 \mu \mathrm{M} \mathrm{ZM449829}$ (Tocris Bioscience) in the RPMI-based complete media containing $10 \%$ FCS for 30 minutes at $37^{\circ} \mathrm{C}$. The inhibitor stock solutions contained DMSO, and thus an equivalent amount of DMSO in media was used as a vehicle control. Optimal inhibitor concentrations were derived from dose-titration experiments using viability, cytotoxicity, and STAT5 phosphorylation (ZM449829 only) as end points (data not shown). For wiskostatin, the minimal concentration inhibiting cytotoxicity was pursued, whereas for ZM449829, the minimal concentration inhibiting STAT5 phosphorylation was targeted. IL-2 (Novartis) at $125 \mathrm{U} / \mathrm{ml}$ in media was used for cell stimulation unless otherwise stated. The concentration was derived from dose-response experiments evaluating cytotoxicity and F-actin content. For measurement of Rac1 activation, 24 hour-serum-starved YTS cells were treated with vehicle or wiskostatin with or without IL-2 prior to cell lysis. When IL-2 was evaluated in inhibitor-treated cells, IL-2 was always added for the specified time after the 30-minute inhibitor pretreatment.

Cytotoxicity. Four-hour ${ }^{51} \mathrm{Cr}$-release assays were performed in round-bottomed 96-well microtiter plates as described (38). YTS, PBMCs, and ex vivo NK cells were used as effectors against 721.221 target cells, or NK92 cells were used as effectors against K562 target cells. The highest effector-to-target cell ratios (E:T) used were 10:1 for YTS and NK92 cells, 20:1 for ex vivo NK cells, and 50:1 for PBMCs. $\mathrm{LU}_{20}$ and $\mathrm{LU}_{20}$ per NK cell were calculated as previously described (16).

Flow cytometry. Flow cytometry was performed for the routine identification of cell subsets among PBMCs and to evaluate purified ex vivo NK cells as described (16). NK cells were defined as $\mathrm{CD} 56^{+} \mathrm{CD}^{-}$and $\mathrm{T}$ cells as $\mathrm{CD}^{+}{ }^{+} \mathrm{CD} 56^{-}$. The $\mathrm{CD} 56^{\text {bright }}$ and $\mathrm{CD} 56^{\mathrm{dim}} \mathrm{NK}$ cell subsets were defined by a gate applied to the nadir between the 2 populations in a histogram analysis of CD56 fluorescence in CD3- cells. Flow cytometry for F-actin content was performed by first incubating cells with antibodies against cell-surface markers for 15 minutes to allow for NK cell identification, followed by permeabilization and fixation using Cytofix/Cytoperm solution (BD Biosciences). Fixed cells were washed 3 times with Perm/Wash solution (BD), incubated for 30 minutes at $4{ }^{\circ} \mathrm{C}$ with Alexa Fluor 647-conjugated phalloidin (Invitrogen), washed 3 times with Perm/Wash, and then fixed in $2 \%$ paraformaldehyde. The percentage change in the MFI in Alexa Fluor 647 phalloidin levels was calculated as follows: $\left(\left[\mathrm{MFI}_{\text {experimental }}-\mathrm{MFI}_{\text {control }}\right] / \mathrm{MFI}_{\text {control }}\right) \times 100$.

Immunoprecipitation and Western blot analysis. For immunoprecipitation, cells were lysed in $25 \mathrm{mM}$ Tris- $\mathrm{Cl}$ ( $\mathrm{pH} 7.5$ ) containing $150 \mathrm{mM} \mathrm{NaCl}$, $5 \mathrm{mM} \mathrm{MgCl}_{2}, 1 \% \mathrm{NP}-40,1 \mathrm{mM} \mathrm{DTT}, 5 \%$ glycerol with protease, and phosphatase inhibitor cocktail (Roche) on ice for 30 minutes. For Western blot analysis of cells only, lysis was performed directly in NuPAGE LDS sample buffer (Invitrogen), directly followed by boiling for 5 minutes prior to gel loading. Proteins were separated on $4 \%-12 \%$ NuPAGE Bis-Tris gels in MOPS SDS running buffer and transferred onto nitrocellulose membranes (Invitrogen). The membranes were blocked in 3\% BSA and incubated with an antibody directed against 1 of the following proteins for 1 hour: WASp (clone 5A5; BD), myosin-IIA (polyclonal M8064; SigmaAldrich), Rac-1 (monoclonal ARC03; Cytoskeleton), STAT5 (polyclonal C-17; Santa Cruz Biotechnology Inc.), phosphoSTAT5 Y694 (clone E208; Abcam), WAVE2 (polyclonal H-110; Santa Cruz Biotechnology Inc.), or phosphoserine (polyclonal Z-PS1; Invitrogen). The membranes were washed, then incubated with secondary antibody for 1 hour, and washed again; bound antibody was detected using ECLplus (Amersham). Where specified, membranes were stripped using $0.2 \mathrm{M}$ glycine ( $\mathrm{pH} 2.5$ ), $0.05 \%$
Tween-20, and $140 \mathrm{mM} \mathrm{NaCl}$ in TRIS-buffered saline at $50^{\circ} \mathrm{C}$ for $30 \mathrm{~min}$ utes prior to blocking and reprobing to detect a different protein. Band intensities were measured using Image J software (NIH Shareware).

For immunoprecipitation, lysates were centrifuged at $14,000 \mathrm{~g}$ and the supernatant precleared with protein $\mathrm{G}$ agarose (Invitrogen) for $30 \mathrm{~min}$ utes at $4^{\circ} \mathrm{C}$ and centrifuged again to remove beads. Cleared supernatant was then incubated at $4^{\circ} \mathrm{C}$ for 1 hour with protein $\mathrm{G}$ agarose that had been incubated overnight with $2 \mu \mathrm{g}$ antibody, centrifuged, washed 3 times in lysis buffer, and centrifuged to separate supernatant and precipitate. Activated Rac1 was precipitated from cell lysates using PAK-1 p21 binding domain GST fusion protein agarose beads (Cytoskeleton).

Cytoskeletal fractionation and analysis of F-actin content. Isolation of insoluble F-actin-rich fractions from cell lysates was performed as described (39). Briefly, $1 \times 10^{7}$ YTS cells expressing GFP-actin were either left unstimulated in R-10 media or stimulated by the addition of $125 \mathrm{U} / \mathrm{ml} \mathrm{IL-2.} \mathrm{Cells}$ were collected by centrifugation, lysed in cytoskeletal stabilizing lysis buffer (80 mM PIPES [pH 6.9], 1\% Triton X-100, 1 mM EGTA, 1 mM MgCl , HALT protease inhibitor cocktail [Pierce]), and centrifuged at $5000 \mathrm{~g}$ for 5 minutes. The F-actin-rich pellet, also containing nuclei, was resuspended in high-salt buffer (20 mM HEPES [pH 7.9], $0.4 \mathrm{M} \mathrm{NaCl}, 1 \mathrm{mM}$ EDTA, HALT protease inhibitor cocktail) and vortexed for 1 hour at $4^{\circ} \mathrm{C}$ to extract proteins. Samples were subjected to centrifugation at $16,000 \mathrm{~g}$ for $10 \mathrm{~min}$ utes, and supernatants representing $2 \times 10^{6}$ cell equivalents were used for Western blot analysis with anti-actin (polyclonal A5060; Sigma-Aldrich) or anti-GFP antibodies (monoclonal 9996; Santa Cruz Biotechnology Inc.), thus allowing distinction of GFP-actin and endogenous actin. Endogenous and GFP-actin were additionally distinguished by molecular weight difference. In addition to analysis of GFP-actin and endogenous F-actin content, the nuclear protein histone $\mathrm{H} 1$ was used as a loading control (with monoclonal 8030; Santa Cruz Biotechnology Inc.) for F-actin-rich fractions.

Microscopy and image analysis. Cells were prepared for evaluation of fixed conjugates by immunoflourescent microscopy as previously described (37). Live cell confocal microscopy was performed as described previously (38) but employed YTS cells stably expressing human actin fused to GFP (GFPactin). These cells were generated via transduction of a retroviral vector (provided by Janis Burkardt, Children's Hospital of Philadelphia) as previously reported (37). To evaluate GFP-actin redistribution, cells were loaded with $5 \mu \mathrm{M}$ DRAQ5 (Biostatus LTD) for 5 minutes at room temperature to allow nuclear visualization, washed, and added to an environmentally controlled imaging chamber (Bioptechs $\Delta \mathrm{T}$ system) in $0.5 \mathrm{ml}$ media; after cells were stably visualized in the field of view, IL-2 or vehicle was added to the chamber media. TIRF microscopy was performed as described (38), but using YTS-GFP or YTS-GFP-actin cells adhered to glass coverslips precoated with anti-CD18 (clone TS1.18.1; ATCC) and anti-NKp30 (clone Z25; Beckman Coulter). These were stained with phalloidin prior to fixation on the slides as per the fixed cell conjugates. Microscopy was performed using a spinning-disc confocal, TIRF microscope (Olympus IX81 with DSU) employing a $\times 601.49$ NA objective and a Hamamatsu EM-CCD camera. Illumination was provided for confocal microscopy using an Olympus $100 \mathrm{~W}$ halogen source with narrow bandpass filters (Chroma) and for TIRF via 488- and 568-nm lasers through an LMM5 laser combiner unit (Spectral Imaging). For some experiments, a Zeiss (Axio observer Z1) fluorescence microscope was used, which was outfitted with a $\times 631.42$ NA objective and Yokogawa CSU-10 spinning disc with illumination provided from 405-nm, 488-nm, 568-nm, and 655-nm lasers in a LMM5 laser combiner unit (Spectral).

Images were acquired using Volocity (PerkinElmer) acquisition and analyzed using the Volocity classification module. Previously described and validated algorithms were utilized to measure F-actin accumulation (23) and perforin distance from the IS (37). GFP margination in live cells was measured by comparing the MFI of the cell footprint to 
the brightest cortical regions using a 2SD classifier with the following formula: $\%$ change $=\left(\left[\mathrm{MFI}_{\text {cortical }}-\mathrm{MFI}_{\text {footprint }}\right] / \mathrm{MFI}_{\text {footprint }}\right) \times 100$. Colocalization analysis in TIRF images was performed as described (38). Pixel intensity plots and correlation coefficients were generated using the colocalization tool in Volocity.

For transmission electron microscopy, YTS cells were activated for 30 minutes on glass cover slips coated with $\alpha$-CD28 (clone 9.3) and $\alpha$-CD11a (clone TS1/22). Cover slips were rinsed in PBS, immersed for 10 seconds into 1/3× PEM buffer (0.1 M PIPES [Sigma-Aldrich], pH 6.9, 1 mM EGTA [Sigma-Aldrich], $1 \mathrm{mM} \mathrm{MgCl} 2$ [Sigma-Aldrich]), sonicated in PEM, incubated for 1-2 minutes in 1\% Triton X-100 in PEM, and fixed in 2\% glutaraldehyde in PEM. Platinum replicas were made as described (40) and imaged on a JEM 1011 transmission electron microscope (JEOL USA) with an ORIUS 835.10W CCD camera (Gatan).

In vivo administration of IL-2. The protocol for administration of IL-2 (ClinicalTrials.gov identifier NCT00774358) was approved by the Internal Review Board for the Protection of Human Subjects of the Children's Hospital of Philadelphia (protocol number 2007-6-5354) and is in accordance with an investigational new drug approval (\#101559) from the US Food and Drug Administration for a phase I clinical trial. While this particular trial is ongoing, the experience from the first enrollment is presented for proof of concept. A 13-year-old patient with a $134 \mathrm{C}>\mathrm{T}$ WASP mutation predicting a T45M missense alteration previously associated with $80 \%$ reduction in WASp levels (22) was enrolled in the protocol after assent and parental consent. The patient had grade- 2 WAS according to the WAS severity scale (41) and had persistent thrombocytopenia, recurrent respiratory tract infection, and a family history of maternal uncles who died prematurely from hematologic malignancy. After screening, IL-2 (Novartis) was administered subcutaneously at $0.5 \times 10^{6} \mathrm{U} / \mathrm{m}^{2}$ daily for 5 days. Blood samples were taken before, immediately after, and at approximately 2 -week intervals after the treatment course so that monitoring laboratory tests and immunologic assessments could be performed. The treatment was repeated 9 and 19 weeks after the first and the protocol concluded at the end of 27 weeks. While pharmacokinetic assessments were not possible due to blood volume limitations, a similar subcutaneous dosing regimen has been evaluated in a previous study that identified a $\mathrm{T}_{\max }$ and half-life for IL-2 of approximately 4 hours (26). Others have defined serum concentrations of doses similar to those used in the present work to provide serum concentrations in the 77-pM range (42), which are greater than or similar to those found in individuals undergoing certain immune responses, such as mononucleosis $(43,44)$.

siRNA. WAVE2-specific or control siRNA (Santa Cruz Biotechnology Inc.) was nucleofected into YTS cells (program T-20 with reagent R; Amaxa) or ex vivo NK cells (program U-O1 with human NK cell Nucleofector kit; Amaxa). Nucleofection utilized $750 \mathrm{nM}$ siRNA per $5 \times 10^{6}$ cells in a single cuvette followed by 24 hours incubation in $10 \%$ FCS-containing media at $37^{\circ} \mathrm{C}$ prior to use.

Statistics. Data were compared using Student's 2-tailed $t$ test or 2-tailed Mann-Whitney $U$ test (where specified), and significance defined as $P<0.05$.

\section{Acknowledgments}

The authors thank the following individuals for support of patient treatment: P. Adamson, L. Speicher, K. Nichols, J. Burnham, A. Cianferoni, M. Cohen, and P. Gallagher as well as D. Depaul, and G. Friedberger. This work was supported by an NIH grant (R01 AI-067946 to J.S. Orange), the University of Pennsylvania School of Medicine and Children's Hospital of Philadelphia Clinical and Translational Science Award (UL1-RR024134) from the National Center for Research Resources through the Clinical and Translational Research Center (support to J.S. Orange) and Junior Investigator Grants Program (to S. Roy-Ghanta), the Institute for Translational Medicine and Therapeutics (ITMAT) Transdisciplinary Program in Translational Medicine and Therapeutics (support to J.S. Orange), the Jeffrey Modell Foundation, and the Children's Hospital of Philadelphia Research Institute Foundation. The content is solely the responsibility of the authors and does not represent official views of the National Center for Research Resources or the NIH. The authors are grateful to the research subject and his family for their participation in this study.

Received for publication August 21, 2010, and accepted in revised form January 12, 2011.

Address correspondence to: Jordan S. Orange, 3615 Civic Center Blvd., ARC 907A, Philadelphia, Pennsylvania 19104, USA. Phone: 267.426.5622; Fax: 267.426.5727; E-mail: orange@upenn.edu.
1. Nolz JC, et al. The WAVE2 complex regulates actin cytoskeletal reorganization and CRAC-mediated calcium entry during $\mathrm{T}$ cell activation. Curr Biol. 2006;16(1):24-34.

2. Nolz JC, et al. The WAVE2 complex regulates T cell receptor signaling to integrins via Abl- and CrkL-C3G-mediated activation of Rap1.J Cell Biol. 2008;182(6):1231-1244.

3. Zhang J, et al. Antigen receptor-induced activation and cytoskeletal rearrangement are impaired in Wiskott-Aldrich syndrome protein-deficient lymphocytes. J Exp Med. 1999;190(9):1329-1342.

4. Cannon JL, Burkhardt JK. Differential roles for Wiskott-Aldrich syndrome protein in immune synapse formation and IL-2 production. J Immunol. 2004;173(3):1658-1662.

5. Kenney D, Cairns L, Remold-O’Donnell E, Peterson J, Rosen FS, Parkman R. Morphological abnormalities in the lymphocytes of patients with the WiskottAldrich syndrome. Blood. 1986;68(6):1329-1332.

6. Trifari S, et al. Defective Th1 cytokine gene transcription in CD4+ and CD8+ T cells from Wiskott-Aldrich syndrome patients. J Immunol. 2006; 177(10):7451-7461.

7. Molina IJ, Sancho J, Terhorst C, Rosen FS, Remold-O'Donnell E. T cells of patients with the Wiskott-Aldrich syndrome have a restricted defect in proliferative responses. J Immunol. 1993;151(8):4383-4390.

8. Strom TS, Gabbard W, Kelly PF, Cunningham JM, Nienhuis AW. Functional correction of T cells derived from patients with the Wiskott-Aldrich syndrome (WAS) by transduction with an oncoretroviral vector encoding the WAS protein. Gene Ther. 2003;10(9):803-809.

9. Lopez-Botet M, Fontan G, Garcia Rodriguez MC, de Landazuri MO. Relationship between IL 2 synthesis and the proliferative response to PHA in different primary immunodeficiencies. J Immunol. 1982;128(2):679-683.

10. Sullivan KE, Mullen CA, Blaese RM, Winkelstein JA. A multiinstitutional survey of the Wiskott-Aldrich syndrome. J Pediatr. 1994;125(6 pt 1):876-885.

11. Orange JS. Human natural killer cell deficiencies and susceptibility to infection. Microbes Infect. 2002;4(15):1545-1558.

12. Kuijpers TW, Baars PA, Dantin C, van den Burg M, van Lier RA, Roosnek E. Human NK cells can control CMV infection in the absence of T cells. Blood. 2008;112(3):914-915.

13. Waldhauer I, Steinle A. NK cells and cancer immunosurveillance. Oncogene. 2008;27(45):5932-5943.

14. Orange JS. Formation and function of the lytic NK-cell immunological synapse. Nat Rev Immunol.
2008;8(9):713-725.

15. Orange JS, Harris KE, Andzelm MM, Valter MM, Geha RS, Strominger JL. The mature activating natural killer cell immunologic synapse is formed in distinct stages. Proc Natl Acad Sci U S A. 2003;100(24):14151-14156.

16. Orange JS, et al. Wiskott-Aldrich syndrome protein is required for $\mathrm{NK}$ cell cytotoxicity and colocalizes with actin to NK cell-activating immunologic synapses. Proc Natl Acad Sci U S A. 2002;99(17):11351-11356.

17. Gismondi A, et al. Impaired natural and CD16mediated NK cell cytotoxicity in patients with WAS and XLT: ability of IL-2 to correct NK cell functional defect. Blood. 2004;104(2):436-443.

18. Albert MH, et al. X-linked thrombocytopenia (XLT) due to WAS mutations: clinical characteristics, long-term outcome, and treatment options. Blood. 2010;115(16):3231-3238.

19. De Meester J, Calvez R, Valitutti S, Dupre L. The Wiskott-Aldrich syndrome protein regulates CTL cytotoxicity and is required for efficient killing of B cell lymphoma targets. J Lenkoc Biol. 2010; 88(5):1031-1040.

20. Yodoi J, et al. TCGF (IL 2)-receptor inducing factor(s). I. Regulation of IL 2 receptor on a natural killer-like cell line (YT cells). J Immunol. 1985; 134(3):1623-1630. 
21. Gong JH, Maki G, Klingemann HG. Characterization of a human cell line (NK-92) with phenotypi$\mathrm{cal}$ and functional characteristics of activated natural killer cells. Leukemia. 1994;8(4):652-658.

22. Lutskiy MI, Rosen FS, Remold-O’Donnell E. Genotype-proteotype linkage in the Wiskott-Aldrich syndrome. J Immunol. 2005;175(2):1329-1336.

23. Banerjee PP, Orange JS. Quantitative measurement of F-actin accumulation at the NK cell immunological synapse. J Immunol Methods. 2010;355(1-2):1-13.

24. Peterson JR, et al. Chemical inhibition of N-WASP by stabilization of a native autoinhibited conformation. Nat Struct Mol Biol. 2004;11(8):747-755.

25. Gross ER, Hsu AK, Gross GJ. The JAK/STAT pathway is essential for opioid-induced cardioprotection:JAK2 as a mediator of STAT3, Akt, and GSK-3 beta. Am J Physiol Heart Circ Physiol. 2006;291(2):H827-H834.

26. Piscitelli SC, Wells MJ, Metcalf JA, Baseler M, Stevens R, Davey RT Jr. Pharmacokinetics and pharmacodynamics of subcutaneous interleukin-2 in HIV-infected patients. Pharmacotherapy. 1996; 16(5):754-759.

27. Baume DM, Robertson MJ, Levine H, Manley TJ, Schow PW, Ritz J. Differential responses to interleukin 2 define functionally distinct subsets of human natural killer cells. Eur J Immunol. 1992;22(1):1-6.

28. Lebensohn AM, Kirschner MW. Activation of the WAVE complex by coincident signals controls actin assembly. Mol Cell. 2009;36(3):512-524.

29. Danson CM, Pocha SM, Bloomberg GB, Cory GO. Phosphorylation of WAVE2 by MAP kinases regu- lates persistent cell migration and polarity. $J$ Cell Sci. 2007;120(pt 23):4144-4154.

30. Nolz JC, et al. WAVE2 regulates high-affinity integrin binding by recruiting vinculin and talin to the immunological synapse. Mol Cell Biol. 2007;27(17):5986-6000.

31. Huang Y, Comiskey EO, Dupree RS, Li S, Koleske AJ, Burkhardt JK. The c-Abl tyrosine kinase regulates actin remodeling at the immune synapse. Blood. 2008;112(1):111-119.

32. Gallego MD, Santamaria M, Pena J, Molina IJ. Defective actin reorganization and polymerization of Wiskott-Aldrich T cells in response to CD3-mediated stimulation. Blood. 1997;90(8):3089-3097.

33. Cianferoni A, et al. Defective nuclear translocation of nuclear factor of activated $T$ cells and extracellular signal-regulated kinase underlies deficient IL-2 gene expression in Wiskott-Aldrich syndrome. J Allergy Clin Immunol. 2005;116(6):1364-1371.

34. Azuma H, Sakata H, Saijyou M, Okuno A. Effect of interleukin 2 on intractable herpes virus infection and chronic eczematoid dermatitis in a patient with Wiskott-Aldrich syndrome. Eur J Pediatr. 1993;152(12):998-1000.

35. Starr SE, et al. Phase I/II trial of intravenous recombinant interleukin-2 in HIV-infected children. Aids. 2003;17(15):2181-2189.

36. Azuma $\mathrm{H}$, et al. Impaired interleukin-2 production in T-cells from a patient with Wiskott-Aldrich syndrome: basis of clinical effect of interleukin-2 replacement therapy. Eur J Pediatr. 2000;159(8):633-634.

37. Banerjee PP, Pandey R, Zheng R, Suhoski MM,
Monaco-Shawver L, Orange JS. Cdc42-interacting protein-4 functionally links actin and microtubule networks at the cytolytic NK cell immunological synapse. J Exp Med. 2007;204(10):2305-2320.

38. Sanborn KB, et al. Myosin IIA associates with NK cell lytic granules to enable their interaction with F-actin and function at the immunological synapse. J Immunol. 2009;182(11):6969-6984.

39. Carrizosa E, et al. Hematopoietic lineage cell-specific protein 1 is recruited to the immunological synapse by IL-2-inducible T cell kinase and regulates phospholipase Cgamma1 Microcluster dynamics during $\mathrm{T}$ cell spreading. J Immunol. 2009;183(11):7352-7361.

40. Svitkina T. Electron microscopic analysis of the leading edge in migrating cells. Methods Cell Biol. 2007;79:295-319.

41. Zhu Q, et al. Wiskott-Aldrich syndrome/X-linked thrombocytopenia: WASP gene mutations, protein expression, and phenotype. Blood. 1997; 90(7):2680-2689

42. Caligiuri MA, et al. Selective modulation of human natural killer cells in vivo after prolonged infusion of low dose recombinant interleukin 2.J Clin Invest. 1993;91(1):123-132.

43. Hornef MW, Wagner HJ, Kruse A, Kirchner H. Cytokine production in a whole-blood assay after Epstein-Barr virus infection in vivo. Clin Diagn Lab Immunol. 1995;2(2):209-213.

44. Wright-Browne V, et al. Serum cytokine levels in infectious mononucleosis at diagnosis and convalescence. Leuk Lymphoma. 1998;30(5-6):583-589. 